\title{
Right Bregman Nonexpansive Operators in Banach Spaces
}

\author{
Victoria Martín-Márquez, Simeon Reich ${ }^{\dagger}$ and Shoham Sabach ${ }^{\ddagger}$ \\ May 15, 2012
}

\begin{abstract}
We introduce and study new classes of Bregman nonexpansive operators in reflexive Banach spaces. These classes of operators are associated with the Bregman distance induced by a convex function. In particular, we characterize sunny right quasi-Bregman nonexpansive retractions, and as a consequence, we show that the fixed point set of any right quasi-Bregman nonexpansive operator is a sunny right quasi-Bregman nonexpansive retract of the ambient Banach space.
\end{abstract}

2010 Mathematics Subject Classification: 26B25, 46T99, 47H04, 47H05, 47H09, 47H10, 52A41, 54C15.

Key words and phrases. Boltzmann-Shannon entropy, Bregman distance, Bregman firmly nonexpansive operator, Fermi-Dirac entropy, Legendre function, monotone mapping, nonexpansive operator, reflexive Banach space, resolvent, retraction, T-monotone mapping, totally convex function.

*Departamento de Análisis Matemático, Universidad de Sevilla, Apdo. 1160, 41080-Sevilla, Spain. Email: victoriam@us.es.

$\dagger$ Department of Mathematics, The Technion - Israel Institute of Technology, 32000 Haifa, Israel. Email: sreich@tx.technion.ac.il.

${ }^{\ddagger}$ Department of Mathematics, The Technion - Israel Institute of Technology, 32000 Haifa, Israel. Email: ssabach@tx.technion.ac.il. 


\section{Introduction}

Nonexpansive operator theory in Banach spaces has been an important topic in Nonlinear Functional Analysis and Optimization Theory for almost fifty years now [5, 24, 25, 26]. There are several significant classes of nonexpansive operators which enjoy remarkable properties not shared by all such operators. We refer, for example, to firmly and strongly nonexpansive operators $[15,16]$. In particular, firmly nonexpansive operators are of utmost importance in Fixed Point, Monotone Mapping, and Convex Optimization Theories in view of Minty's Theorem regarding the correspondence between firmly nonexpansive operators and maximal monotone mappings [5, 24, 26, 33]. The class of strongly nonexpansive operators, which embraces firmly nonexpansive operators, presents the advantage of its being closed under compositions, whereas this property fails for firmly nonexpansive mappings [16, 34]. A related class of operators comprises the quasi-nonexpansive operators. These operators still enjoy relevant fixed point properties although nonexpansivity is only required for each fixed point [22].

A basic example of a firmly nonexpansive operator is the nearest point projection onto a closed and convex subset of a Hilbert space. It is characterized by a certain kind of quasi-firm nonexpansivity. An extension of this nonexpansivity property to general smooth Banach spaces characterizes the unique so-called sunny nonexpansive retraction [15, 32]. For this reason, sunny nonexpansive retractions seem to play a similar role in Banach spaces to that of nearest point projections in Hilbert spaces. More information concerning (sunny) nonexpansive retractions and their applications can be found, for example, in [26, 28] and the references therein.

In this paper, we are concerned with certain analogous classes of operators which are, in some sense, nonexpansive not with respect to the norm, but with respect to Bregman distances $[13,18,21]$. Since these distances are not symmetric in general, it seems natural to distinguish between left and right Bregman nonexpansive operators. Some left classes, so to speak, have already been studied quite intensively [4, 8, 29, 35]. In the present paper, we concentrate on the right ones.

Our paper is organized as follows. In Section 2 we discuss several pertinent facts of Convex Analysis and Bregman Operator Theory. In Section 3, we investigate the properties of several classes of right Bregman nonexpansive operators. Section 4 is devoted to right Bregman nonexpansive retractions. We show there that the fixed point set of any right quasi-Bregman nonexpansive operator is a sunny right quasi-Bregman nonexpansive retract of the ambient Banach space (Corollary 4.7). This property of the right Bregman nonexpansive operators, not enjoyed by their left counterparts, is an analogue of a classical result of Bruck's [15]. We also point out 
that the corresponding right Bregman projection is consequently the unique sunny right quasi-Bregman nonexpansive retraction, while the left Bregman projection is not sunny, but has a sunny-like property. In Section 5, we study the conjugate resolvent and provide a characterization of right Bregman firmly nonexpansive operators (Theorem 5.6). Finally, in Section 6, we present many examples of right Bregman firmly nonexpansive operators.

\section{Preliminaries}

All the results in this paper are set in a real reflexive Banach space $X$. The norms of $X$ and $X^{*}$, its dual space, are denoted by $\|\cdot\|$ and $\|\cdot\|_{*}$, respectively. The pairing $\langle\xi, x\rangle$ is defined by the action of $\xi \in X^{*}$ at $x \in X$, that is, $\langle\xi, x\rangle:=\xi(x)$. The set of all real numbers is denoted by $\mathbb{R}$ and $\overline{\mathbb{R}}=(-\infty,+\infty]$ is the extended real line, while $\mathbb{N}$ stands for the set of nonnegative integers. The closure of a subset $K$ of $X$ is denoted by $\bar{K}$. The domain of a convex function $f: X \rightarrow \overline{\mathbb{R}}$ is defined to be

$$
\operatorname{dom} f:=\{x \in X: f(x)<+\infty\}
$$

When $\operatorname{dom} f \neq \emptyset$ we say that $f$ is proper. The Fenchel conjugate function of $f$ is the convex function $f^{*}: X^{*} \rightarrow \overline{\mathbb{R}}$ defined by

$$
f^{*}(\xi)=\sup \{\langle\xi, x\rangle-f(x): x \in X\}
$$

It is not difficult to check that when $f$ is proper and lower semicontinuous, so is $f^{*}$. The function $f$ is said to be cofinite if $\operatorname{dom} f^{*}=X^{*}$.

In this section, we present the basic notions and facts that are needed in the sequel. We divide this section into three parts in the following way. The first one (Subsection 2.1) is devoted to admissible functions, while the second and the third (Subsections 2.2 and 2.3) concern certain types of Bregman nonexpansive operators and monotone mappings, respectively.

\subsection{Admissible functions}

Let $x \in \operatorname{int} \operatorname{dom} f$, that is, let $x$ belong to the interior of the domain of the convex function $f: X \rightarrow \overline{\mathbb{R}}$. For any $y \in X$, we define the directional derivative of $f$ at $x$ by

$$
f^{\circ}(x, y):=\lim _{t \rightarrow 0^{+}} \frac{f(x+t y)-f(x)}{t} .
$$


If the limit as $t \rightarrow 0$ in (1) exists for each $y$, then the function $f$ is said to be Gâteaux differentiable at $x$. In this case, the gradient of $f$ at $x$ is the linear function $\nabla f(x)$, which is defined by $\langle\nabla f(x), y\rangle:=f^{\circ}(x, y)$ for all $y \in X$ [31, Definition 1.3, page 3]. The function $f$ is called Gâteaux differentiable if it is Gâteaux differentiable at each $x \in \operatorname{int} \operatorname{dom} f$. When the limit as $t \rightarrow 0$ in (1) is attained uniformly for any $y \in X$ with $\|y\|=1$, we say that $f$ is Fréchet differentiable at $x$.

Throughout this paper, $f: X \rightarrow \overline{\mathbb{R}}$ is always an admissible function, that is, a proper, lower semicontinuous, convex and Gâteaux differentiable function. Under these conditions we know that $f$ is continuous in int $\operatorname{dom} f$ (see [3, Fact 2.3, page $619])$.

The function $f$ is said to be Legendre if it satisfies the following two conditions.

(L1) int $\operatorname{dom} f \neq \emptyset$ and the subdifferential $\partial f$ is single-valued on its domain.

(L2) int $\operatorname{dom} f^{*} \neq \emptyset$ and $\partial f^{*}$ is single-valued on its domain.

The class of Legendre functions in infinite dimensional Banach spaces was first introduced and studied by Bauschke, Borwein and Combettes in [3]. Their definition is equivalent to conditions (L1) and (L2) because the space $X$ is assumed to be reflexive (see [3, Theorems 5.4 and 5.6, page 634]). It is well known that in reflexive spaces $\nabla f=\left(\nabla f^{*}\right)^{-1}$ (see $[9$, page 83$\left.]\right)$. When this fact is combined with conditions (L1) and (L2), we obtain

$$
\operatorname{ran} \nabla f=\operatorname{dom} \nabla f^{*}=\operatorname{int} \operatorname{dom} f^{*} \text { and } \operatorname{ran} \nabla f^{*}=\operatorname{dom} \nabla f=\operatorname{int} \operatorname{dom} f .
$$

It also follows that $f$ is Legendre if and only if $f^{*}$ is Legendre (see [3, Corollary 5.5, page 634]) and that the functions $f$ and $f^{*}$ are Gâteaux differentiable and strictly convex in the interior of their respective domains. When the Banach space $X$ is smooth and strictly convex, in particular, a Hilbert space, the function $(1 / p)\|\cdot\|^{p}$ with $p \in(1, \infty)$ is Legendre ( $c f$. [3, Lemma 6.2, page 639]). For examples and more information regarding Legendre functions, see, for instance, $[2,3]$.

The bifunction $D_{f}: \operatorname{dom} f \times \operatorname{int} \operatorname{dom} f \rightarrow[0,+\infty)$, which is defined by

$$
D_{f}(y, x):=f(y)-f(x)-\langle\nabla f(x), y-x\rangle,
$$

is called the Bregman distance ( $c f .[13,20])$.

The Bregman distance does not satisfy the well-known properties of a metric, but it does have the following important property, which is called the three point identity: for any $x \in \operatorname{dom} f$ and $y, z \in \operatorname{int} \operatorname{dom} f$,

$$
D_{f}(x, y)+D_{f}(y, z)-D_{f}(x, z)=\langle\nabla f(z)-\nabla f(y), x-y\rangle \text {. }
$$


According to [18, Section 1.2, page 17] (see also [17]), the modulus of total convexity of $f$ is the bifunction $v_{f}: \operatorname{int} \operatorname{dom} f \times[0,+\infty) \rightarrow[0,+\infty]$ which is defined by

$$
v_{f}(x, t):=\inf \left\{D_{f}(y, x): y \in \operatorname{dom} f,\|y-x\|=t\right\} .
$$

The function $f$ is said to be totally convex at a point $x \in \operatorname{int} \operatorname{dom} f$ if $v_{f}(x, t)>0$ whenever $t>0$. The function $f$ is said to be totally convex when it is totally convex at every point $x \in \operatorname{int} \operatorname{dom} f$. This property is less stringent than uniform convexity (see [18, Section 2.3, page 92]).

Examples of totally convex functions can be found, for instance, in [11, 18, 19]. We remark in passing that $f$ is totally convex on bounded subsets if and only if $f$ is uniformly convex on bounded subsets (see [19, Theorem 2.10, page 9]).

The Bregman projection ( $c f$. [13]) with respect to $f$ of $x \in \operatorname{int} \operatorname{dom} f$ onto a nonempty, closed and convex set $K \subset \operatorname{int} \operatorname{dom} f$ is defined as the necessarily unique vector $\operatorname{proj}_{K}^{f}(x) \in K$, which satisfies

$$
D_{f}\left(\operatorname{proj}_{K}^{f}(x), x\right)=\inf \left\{D_{f}(y, x): y \in K\right\} .
$$

Similarly to the metric projection in Hilbert spaces, the Bregman projection with respect to totally convex and Gâteaux differentiable functions has a variational characterization (cf. [19, Corollary 4.4, page 23]).

Proposition 2.1 (Characterization of Bregman projections). Suppose that $f: X \rightarrow$ $\overline{\mathbb{R}}$ is totally convex and Gâteaux differentiable in $\operatorname{int} \operatorname{dom} f$. Let $x \in \operatorname{int} \operatorname{dom} f$ and let $K \subset \operatorname{int} \operatorname{dom} f$ be a nonempty, closed and convex set. If $\hat{x} \in K$, then the following conditions are equivalent.

(i) The vector $\hat{x}$ is the Bregman projection of $x$ onto $K$ with respect to $f$.

(ii) The vector $\hat{x}$ is the unique solution of the variational inequality

$$
\langle\nabla f(x)-\nabla f(z), z-y\rangle \geq 0 \quad \forall y \in K
$$

(iii) The vector $\hat{x}$ is the unique solution of the inequality

$$
D_{f}(y, z)+D_{f}(z, x) \leq D_{f}(y, x) \quad \forall y \in K \text {. }
$$




\subsection{Bregman nonexpansive operators}

Let $f: X \rightarrow \overline{\mathbb{R}}$ be admissible and let $K$ be a nonempty subset of $X$. The fixed point set of an operator $T: K \rightarrow X$ is the set $\{x \in K: T x=x\}$. It is denoted by Fix $(T)$. Recall that a point $u \in K$ is said to be an asymptotic fixed point [34] of $T$ if there exists a sequence $\left\{x_{n}\right\}_{n \in \mathbb{N}}$ in $K$ such that $x_{n} \rightarrow u$ (that is, $\left\{x_{n}\right\}_{n \in \mathbb{N}}$ is weakly convergent to $u$ ) and $\left\|x_{n}-T x_{n}\right\| \rightarrow 0$ as $n \rightarrow \infty$. We denote the asymptotic fixed point set of $T$ by $\widehat{\operatorname{Fix}}(T)$.

We next list significant types of nonexpansivity with respect to the Bregman distance.

Definition 2.2 (Left Bregman nonexpansivity). Let $K$ and $S$ be nonempty subsets of int $\operatorname{dom} f$ and $\operatorname{dom} f$, respectively. An operator $T: K \rightarrow \operatorname{int} \operatorname{dom} f$ is said to be:

(i) left Bregman firmly nonexpansive (L-BFNE) if

$$
\langle\nabla f(T x)-\nabla f(T y), T x-T y\rangle \leq\langle\nabla f(x)-\nabla f(y), T x-T y\rangle
$$

for any $x, y \in K$, or equivalently,

$$
\begin{aligned}
D_{f}(T x, T y)+D_{f}(T y, T x) & +D_{f}(T x, x)+D_{f}(T y, y) \\
& \leq D_{f}(T x, y)+D_{f}(T y, x) .
\end{aligned}
$$

(ii) Left quasi-Bregman firmly nonexpansive (L-QBFNE) with respect to $S$ if

$$
0 \leq\langle\nabla f(x)-\nabla f(T x), T x-p\rangle
$$

for any $x \in K$ and $p \in S$, or equivalently,

$$
D_{f}(p, T x)+D_{f}(T x, x) \leq D_{f}(p, x) .
$$

(iii) Left quasi-Bregman nonexpansive (L-QBNE) with respect to $S$ if

$$
D_{f}(p, T x) \leq D_{f}(p, x) \forall x \in K, p \in S .
$$

(iv) Left Bregman strongly nonexpansive (L-BSNE) with respect to $S$ if it is LQBNE with respect to $S$ and if whenever $\left\{x_{n}\right\}_{n \in \mathbb{N}} \subset K$ is bounded, $p \in S$, and

$$
\lim _{n \rightarrow \infty}\left(D_{f}\left(p, x_{n}\right)-D_{f}\left(p, T x_{n}\right)\right)=0
$$

it follows that

$$
\lim _{n \rightarrow \infty} D_{f}\left(T x_{n}, x_{n}\right)=0 .
$$


See $[4,11,35]$ for more information and examples of L-BFNE operators (operators in this class are also called $D_{f}$-firm and BFNE). For two recent studies of the existence and approximation of fixed points of left Bregman firmly nonexpansive operators see $[29,35]$.

Recalling that the Bregman distance is not symmetric, and motivated by Definition 2.2(i)-(iv), we define the following operators by "switching places".

Definition 2.3 (Right Bregman nonexpansivity). Let $K$ and $S$ be nonempty subsets of $\operatorname{dom} f$ and int $\operatorname{dom} f$, respectively. An operator $T: K \rightarrow \operatorname{int} \operatorname{dom} f$ is said to be:

(i*) right Bregman firmly nonexpansive (R-BFNE) if

$$
\langle\nabla f(T x)-\nabla f(T y), T x-T y\rangle \leq\langle\nabla f(T x)-\nabla f(T y), x-y\rangle
$$

for any $x, y \in K$, or equivalently,

$$
\begin{aligned}
D_{f}(T x, T y)+D_{f}(T y, T x) & +D_{f}(x, T x)+D_{f}(y, T y) \\
& \leq D_{f}(x, T y)+D_{f}(y, T x) .
\end{aligned}
$$

(ii*) Right quasi-Bregman firmly nonexpansive (R-QBFNE) with respect to $S$ if

$$
0 \leq\langle\nabla f(p)-\nabla f(T x), T x-x\rangle
$$

for any $x \in K$ and $p \in S$, or equivalently,

$$
D_{f}(T x, p)+D_{f}(x, T x) \leq D_{f}(x, p) .
$$

(iii*) Right quasi-Bregman nonexpansive (R-QBNE) with respect to $S$ if

$$
D_{f}(T x, p) \leq D_{f}(x, p) \forall x \in K, p \in S \text {. }
$$

(iv*) Right Bregman strongly nonexpansive (R-BSNE) with respect to $S$ if it is RQBNE with respect to $S$ and if whenever $\left\{x_{n}\right\}_{n \in \mathbb{N}} \subset K$ is bounded, $p \in S$, and

$$
\lim _{n \rightarrow \infty}\left(D_{f}\left(x_{n}, p\right)-D_{f}\left(T x_{n}, p\right)\right)=0
$$

it follows that

$$
\lim _{n \rightarrow \infty} D_{f}\left(x_{n}, T x_{n}\right)=0
$$


Remark 2.4 (Types of Bregman nonexpansivity with respect to $S$ ). As in [29], we may distinguish between two types of Bregman nonexpansivity, depending on the set $S$, in such a way that if $S=\operatorname{Fix}(T)$ we say that $T$ is properly Bregman nonexpansive, whereas if $S=\widehat{\operatorname{Fix}}(T)$ we say that $T$ is strictly Bregman nonexpansive, according to the different notions of Bregman nonexpansivity. The connections among all the classes of either left or right Bregman nonexpansive operators are presented in Table 1 .

\begin{tabular}{cccccc|} 
& strictly QBFNE & $\Rightarrow$ & strictly BSNE & $\Rightarrow$ & strictly QBNE \\
$\Downarrow$ & & & $\Downarrow$ & & $\Downarrow$ \\
BFNE $\Rightarrow$ & properly QBFNE & $\Rightarrow$ & properly BSNE & $\Rightarrow$ & properly QBNE \\
\hline
\end{tabular}

Table 1: Connections among types of Bregman nonexpansivity

Throughout this paper, we just deal with these notions of Bregman nonexpansivity with respect to $S=$ Fix $(T)$, so for the sake of simplicity, we omit the prefix "properly" from now on.

Remark 2.5 (Classical notions of nonexpansivity). Let $f=(1 / 2)\|\cdot\|^{2}$ be defined on a Hilbert space $H$. Then $\nabla f=I$ (the identity operator) and $D_{f}(y, x)=$ $(1 / 2)\|x-y\|^{2}$. Thence, Definitions 2.2(i)-(iii) and 2.3(i*)-(iii*) for $S=$ Fix $(T)$ say that the operator $T: K \rightarrow H$ is:

(i') firmly nonexpansive (FNE) if

$$
\|T x-T y\|^{2} \leq\langle x-y, T x-T y\rangle \forall x, y \in K
$$

(ii') Quasi-firmly nonexpansive (QFNE) if

$$
\|T x-p\|^{2}+\|T x-x\|^{2} \leq\langle x-p\rangle^{2}
$$

for any $x \in K, p \in \operatorname{Fix}(T)$, or equivalently,

$$
0 \leq\langle x-T x, T x-p\rangle .
$$

(iii') Quasi-nonexpansive (QNE) if

$$
\|T x-p\| \leq\|x-p\| \forall x \in K, p \in \operatorname{Fix}(T) .
$$


The norm analogue of Bregman strong nonexpansivity (see Definitions 2.2(iv) and $\left.2.3\left(\mathrm{iv}^{*}\right)\right)$ is the notion of strong nonexpansivity, first studied in [16]. We say that $T: K \rightarrow H$ is:

$\left(\mathrm{iv}^{\prime}\right)$ strongly nonexpansive (SNE) if $T$ is nonexpansive and for any bounded sequence $\left\{x_{n}-y_{n}\right\}_{n \in \mathbb{N}}$ satisfying

$$
\lim _{n \rightarrow \infty}\left(\left\|x_{n}-y_{n}\right\|-\left\|T x_{n}-T y_{n}\right\|\right)=0
$$

it follows that

$$
\lim _{n \rightarrow \infty}\left(\left(x_{n}-y_{n}\right)-\left(T x_{n}-T y_{n}\right)\right)=0
$$

The connection between L-BSNE and SNE operators was discussed in [29], and obviously the same connection holds for R-BSNE operators.

If $X$ is a uniformly smooth and uniformly convex Banach space and $f=(1 / 2)\|\cdot\|^{2}$, then $\nabla f=J$, where $J$ is the duality mapping of the space $X$. Hence inequality (12) in Definition $2.3\left(\mathrm{i}^{*}\right)$ becomes

$$
\langle J T x-J T y, T x-T y\rangle \leq\langle J T x-J T y, x-y\rangle
$$

for all $x, y \in K$. An operator which satisfies (25) is called an operator of type $(R)$ (see [1]).

Definition 2.6 (Conjugate operator). Let $f: X \rightarrow \overline{\mathbb{R}}$ be Legendre and $T: K \subset$ $\operatorname{int} \operatorname{dom} f \rightarrow \operatorname{int} \operatorname{dom} f$ be an operator. We define the conjugate operator associated with $T$ by

$$
T_{f}^{*}:=\nabla f \circ T \circ \nabla f^{*}: \nabla f(K) \rightarrow \operatorname{int} \operatorname{dom} f^{*} .
$$

This operator, which was previously used in $[6,8]$, allows us to get connections between left and right Bregman nonexpansive operators, which are collected along with several properties of $T_{f}^{*}$ in the following proposition. Further analysis of the conjugate operator and its usefulness in connecting left and right Bregman nonexpansive operators is provided in [30]. When there is no danger of confusion we use the notation $T^{*}$ for $T_{f}^{*}$. We also denote $\left(T_{f}^{*}\right)_{f^{*}}^{*}$ by $T^{* *}$.

Proposition 2.7 (Properties of the conjugate operator). Let $f: X \rightarrow \overline{\mathbb{R}}$ be Legendre and consider an operator $T: K \subset \operatorname{int} \operatorname{dom} f \rightarrow \operatorname{int} \operatorname{dom} f$. Then the following properties hold. 
(i) $\operatorname{dom} T^{*}=\nabla f(\operatorname{dom} T)$ and $\operatorname{ran} T^{*}=\nabla f(\operatorname{ran} T)$.

(ii) $T$ is $R$-BFNE if and only if $T^{*}$ is $L-B F N E$.

(iii) $\operatorname{Fix}(T)=\nabla f^{*}\left(\operatorname{Fix}\left(T^{*}\right)\right)$.

(iv) $T$ is $R$-QBFNE (R-QBNE or $R$-BSNE) if and only if $T^{*}$ is $L$-QBFNE (L$Q B N E$ or $L-B S N E)$.

(v) $T^{* *}=T$.

(vi) If, in addition, $\nabla f$ and $\nabla f^{*}$ are uniformly continuous on bounded subsets of int $\operatorname{dom} f$ and int $\operatorname{dom} f^{*}$, respectively, then

$$
\widehat{\operatorname{Fix}}\left(T^{*}\right)=\nabla f(\widehat{\operatorname{Fix}}(T)) .
$$

Proof. Since $(\nabla f)^{-1}=\nabla f^{*}$, it is easy to check that

$$
D_{f^{*}}(\nabla f(y), \nabla f(x))=D_{f}(x, y) .
$$

Hence (i) and (iii) can be readily proved. As a consequence, (ii), (iv) and (v) also hold. The proof of (vi) follows from the definition of the asymptotic fixed point set of an operator.

At this point, it would be natural to define the right analog of the classical Bregman projection (hereafter called the left Bregman projection and denoted by $\overleftarrow{\text { proj }}$ ) in the following way. Given a set $K \subset \operatorname{int} \operatorname{dom} f$, the right Bregman projection onto $K$ is the operator $\overrightarrow{\operatorname{proj}}_{K}^{f}$ : int dom $f \rightarrow K$ defined by

$$
\overrightarrow{\operatorname{proj}}_{K}^{f}(x):=\operatorname{argmin}_{y \in K}\left\{D_{f}(x, y)\right\}=\left\{z \in K: D_{f}(x, z) \leq D_{f}(x, y) \forall y \in K\right\}
$$

for each $x \in \operatorname{int} \operatorname{dom} f$. However, since $D_{f}$ is not convex in the second variable, it is not clear a priori that the right Bregman projection is well defined. Fortunately, this difficulty has already been overcome by Bauschke et al. (cf. [8, Proposition 7.1, page 9]), who proved that

$$
\overrightarrow{\operatorname{proj}}_{K}^{f}=\nabla f^{*} \circ \overleftarrow{\operatorname{proj}}_{\nabla f(K)}^{f^{*}} \circ \nabla f
$$

and established several other properties of $\overrightarrow{\operatorname{proj}}_{K}^{f}$. 


\subsection{Monotone mappings}

Let $A: X \rightarrow 2^{X^{*}}$ be a set-valued mapping. Recall that the (effective) domain of the mapping $A$ is the set $\operatorname{dom} A=\{x \in X: A x \neq \emptyset\}$. We say that $A$ is monotone if for any $x, y \in \operatorname{dom} A$ we have

$$
\xi \in A x \text { and } \eta \in A y \quad \Longrightarrow \quad 0 \leq\langle\xi-\eta, x-y\rangle
$$

A monotone mapping $A$ is said to be maximal if the graph of $A$ is not a proper subset of the graph of any other monotone mapping.

Let $K \subset \operatorname{dom} A$ and $T: K \rightarrow X$. We say that a mapping $A$ is monotone with respect to the mapping $T$, or $T$-monotone [11], if

$$
0 \leq\langle\xi-\eta, T x-T y\rangle
$$

for any $x, y \in K$, where $\xi \in A x$ and $\eta \in A y$. Clearly, when $T=I$ the classes of monotone and $I$-monotone operators coincide.

\section{Properties of classes of right Bregman nonex- pansive operators}

Using the analogy between various classes of L-BNE and R-BNE operators we present in this section several properties of R-BNE operators, the analogues of which are already known for L-BNE operators (see [4] for results regarding L-BNE operators).

We begin by recalling the following result regarding the fixed point set of a left Bregman nonexpansive operator ( $c f$. [35, Lemma 15.5, page 307]). Although it was established for L-BFNE operators, the proof also holds for L-QBNE operators.

Proposition 3.1 (Fix $(T)$ of an L-QBNE operator is closed and convex). Let $f$ : $X \rightarrow \overline{\mathbb{R}}$ be a Legendre function, and let $K \subset \operatorname{int} \operatorname{dom} f$ be nonempty, closed and convex. If $T: K \rightarrow \operatorname{int} \operatorname{dom} f$ is an $L$-QBNE operator, then $\mathrm{Fix}(T)$ is closed and convex.

We now present two properties of the fixed point set Fix $(T)$ of R-QBNE operators. We first show that Fix $(T)$ is closed when $f$ is Legendre and Fréchet differentiable.

Proposition 3.2 (Fix (T) of R-QBNE is closed). Let $f: X \rightarrow \overline{\mathbb{R}}$ be a Legendre and Fréchet differentiable function. Let $K$ be a nonempty and closed subset of int $\operatorname{dom} f$, and let $T: K \rightarrow \operatorname{int} \operatorname{dom} f$ be an $R$-QBNE operator. Then $\operatorname{Fix}(T)$ is closed. 
Proof. It is sufficient to consider the case where Fix $(T)$ is nonempty. Let $\left\{x_{n}\right\}_{n \in \mathbb{N}}$ be a sequence in Fix $(T) \subset K$ such that $x_{n} \rightarrow \bar{x}$ as $n \rightarrow \infty$, where $\bar{x} \in K \subset \operatorname{int} \operatorname{dom} f$ because $K$ is assumed to be closed. Since $T$ is R-QBNE, it follows from (16) that

$$
D_{f}\left(T \bar{x}, x_{n}\right) \leq D_{f}\left(\bar{x}, x_{n}\right)
$$

for each $n \in \mathbb{N}$. Since $f$ is Fréchet differentiable, the gradient $\nabla f$ is norm-to-norm continuous (see [31, Proposition 2.8, page 19]). Since $f$ is continuous in int $\operatorname{dom} f$ and $x_{n} \rightarrow \bar{x}$ as $n \rightarrow \infty$, it now follows that

$$
\begin{aligned}
\lim _{n \rightarrow \infty} D_{f}\left(T \bar{x}, x_{n}\right) & =\lim _{n \rightarrow \infty}\left[f(T \bar{x})-f\left(x_{n}\right)-\left\langle\nabla f\left(x_{n}\right), T \bar{x}-x_{n}\right\rangle\right] \\
& =[f(T \bar{x})-f(\bar{x})-\langle\nabla f(\bar{x}), T \bar{x}-\bar{x}\rangle]=D_{f}(T \bar{x}, \bar{x}) .
\end{aligned}
$$

On the other hand, replacing $T \bar{x}$ with $\bar{x}$, one gets

$$
\lim _{n \rightarrow \infty} D_{f}\left(\bar{x}, x_{n}\right)=D_{f}(\bar{x}, \bar{x})=0 .
$$

Thus (31) implies that $D_{f}(T \bar{x}, \bar{x})=0$ and therefore it follows from [3, Lemma 7.3(vi), page 642] that $\bar{x}=T \bar{x}$. Hence $\bar{x} \in \operatorname{Fix}(T)$ and this means that $\operatorname{Fix}(T)$ is closed, as claimed.

We next prove that $\nabla f(\operatorname{Fix}(T))$ is closed and convex when $T$ is R-QBNE, and $f$ is Legendre and cofinite.

Proposition 3.3 $(\nabla f(\operatorname{Fix}(T))$ of an R-QBNE operator is closed and convex). Let $f: X \rightarrow \overline{\mathbb{R}}$ be a Legendre and cofinite function, and let $T: \operatorname{int} \operatorname{dom} f \rightarrow \operatorname{int} \operatorname{dom} f$ be an $R$-QBNE operator. Then $\nabla f(\operatorname{Fix}(T))$ is a closed and convex subset of $X^{*}$.

Proof. We first show that $\nabla f(\operatorname{Fix}(T))$ is convex. Let $x, y \in \operatorname{Fix}(T), t \in(0,1)$, and set $s=1-t$. If we denote $z=\nabla f^{*}(t \nabla f(x)+s \nabla f(y))$, it is sufficient to prove that $z \in \operatorname{Fix}(T)$. Indeed from the definition of the Bregman distance (see (2)) it follows that

$$
\begin{aligned}
D_{f}(T z, z) & =f(T z)-f(z)-\langle\nabla f(z), T z-z\rangle \\
& =t[f(T z)-f(x)-\langle\nabla f(x), T z-x\rangle] \\
& +s[f(T z)-f(y)-\langle\nabla f(y), T z-y\rangle] \\
& +t f(x)-t\langle\nabla f(x), x\rangle+s f(y)-s\langle\nabla f(y), y\rangle \\
& +\langle\nabla f(z), z\rangle-f(z) \\
& =t D_{f}(T z, x)+s D_{f}(T z, y)+t(f(x)-\langle\nabla f(x), x\rangle) \\
& +s(f(y)-\langle\nabla f(y), y\rangle)-(f(z)-\langle\nabla f(z), z\rangle) .
\end{aligned}
$$


It is known that $f(x)+f^{*}(\nabla f(x))=\langle\nabla f(x), x\rangle$. Therefore

$$
\begin{aligned}
D_{f}(T z, z) & =t D_{f}(T z, x)+s D_{f}(T z, y)-t f^{*}(\nabla f(x)) \\
& -s f^{*}(\nabla f(y))+f^{*}(\nabla f(z)) .
\end{aligned}
$$

Since $T$ is R-QBNE and $x, y \in \operatorname{Fix}(T)$, we see that

$$
\begin{aligned}
D_{f}(T z, z) & \leq t D_{f}(z, x)+s D_{f}(z, y)-t f^{*}(\nabla f(x)) \\
& -s f^{*}(\nabla f(y))+f^{*}(\nabla f(z)) \\
& =t[f(z)-f(x)-\langle\nabla f(x), z-x\rangle]+ \\
& s[f(z)-f(y)-\langle\nabla f(y), z-y\rangle] \\
& -t f^{*}(\nabla f(x))-s f^{*}(\nabla f(y))+f^{*}(\nabla f(z)) \\
& =t\left[\langle\nabla f(x), x\rangle-f(x)-f^{*}(\nabla f(x))\right] \\
& +s\left[\langle\nabla f(y), y\rangle-f(y)-f^{*}(\nabla f(y))\right] \\
& +f(z)+f^{*}(\nabla f(z))-t\langle\nabla f(x), z\rangle-s\langle\nabla f(y), z\rangle \\
& =f(z)+f^{*}(\nabla f(z))-\langle t \nabla f(x)+s \nabla f(y), z\rangle \\
& =f(z)+f^{*}(\nabla f(z))-\langle\nabla f(z), z\rangle=0 .
\end{aligned}
$$

It now follows from [3, Lemma $7.3(\mathrm{vi})$, page 642$]$ that $T z=z$. That is, $z \in \operatorname{Fix}(T)$. Therefore $\nabla f(\operatorname{Fix}(T))$ is convex, as claimed.

Now we prove that $\nabla f(\operatorname{Fix}(T))$ is closed. Let $\left\{x_{n}\right\}_{n \in \mathbb{N}}$ be a sequence in Fix $(T)$ such that $\nabla f\left(x_{n}\right) \rightarrow \xi \in X^{*}$ as $n \rightarrow \infty$. Since $f$ is cofinite, $\operatorname{ran} \nabla f=\operatorname{dom} \nabla f^{*}=$ int dom $f^{*}=X^{*}$. Hence there exists $x \in X$ such that $\xi=\nabla f(x)$. It is sufficient to prove that $x \in \operatorname{Fix}(T)$. Since $\left\{x_{n}\right\}_{n \in \mathbb{N}} \subset \operatorname{Fix}(T)$ and $T$ is R-QBNE, we have

$$
\begin{aligned}
D_{f}\left(T x, x_{n}\right) & \leq D_{f}\left(x, x_{n}\right)=f(x)-f\left(x_{n}\right)-\left\langle\nabla f\left(x_{n}\right), x-x_{n}\right\rangle \\
& =f(x)-\left\langle\nabla f\left(x_{n}\right), x\right\rangle+f^{*}\left(\nabla f\left(x_{n}\right)\right) .
\end{aligned}
$$

By assumption, $f^{*}$ is continuous and $\nabla f\left(x_{n}\right) \rightarrow \nabla f(x)$ as $n \rightarrow \infty$. Hence

$$
\begin{aligned}
\lim _{n \rightarrow \infty} D_{f}\left(T x, x_{n}\right) & \leq \lim _{n \rightarrow \infty}\left(f(x)-\left\langle\nabla f\left(x_{n}\right), x\right\rangle+f^{*}\left(\nabla f\left(x_{n}\right)\right)\right) \\
& =f(x)-\lim _{n \rightarrow \infty}\left(\left\langle\nabla f\left(x_{n}\right), x\right\rangle+f^{*}\left(\nabla f\left(x_{n}\right)\right)\right) \\
& =f(x)-\langle\nabla f(x), x\rangle+f^{*}(\nabla f(x))=0 .
\end{aligned}
$$

On the other hand,

$$
\begin{aligned}
D_{f}(T x, x) & =f(T x)-f(x)-\langle\nabla f(x), T x-x\rangle \\
& =D_{f}\left(T x, x_{n}\right)+f\left(x_{n}\right)-f(x)+\left\langle\nabla f\left(x_{n}\right), T x-x_{n}\right\rangle-\langle\nabla f(x), T x-x\rangle \\
& =D_{f}\left(T x, x_{n}\right)-f^{*}\left(\nabla f\left(x_{n}\right)\right)+f^{*}(\nabla f(x))+\left\langle\nabla f\left(x_{n}\right)-\nabla f(x), T x\right\rangle .
\end{aligned}
$$


Therefore, taking the limit as $n$ tends to $\infty$, we obtain that $D_{f}(T x, x)=0$. It now follows from [3, Lemma $7.3(\mathrm{vi})$, page 642$]$ that $T x=x$. That is, $x \in \operatorname{Fix}(T)$, as claimed. This completes the proof.

Now we present another proof of this fact by using the conjugate operator.

Proposition $3.4(\nabla f(\mathrm{Fix}(T))$ of an R-QBNE operator is closed and convex). Let $f: X \rightarrow \overline{\mathbb{R}}$ be a Legendre function and let $K$ be a nonempty subset of int dom $f$ such that $\nabla f(K)$ is closed and convex. If $T: K \rightarrow \operatorname{int} \operatorname{dom} f$ is an $R$-QBNE operator, then $\nabla f(\operatorname{Fix}(T))$ is closed and convex.

Proof. Since $T$ is R-QBNE, the conjugate operator $T^{*}$ is L-QBNE with respect to $f^{*}$ (see Proposition 2.7(iv)). Moreover, $f^{*}$ is Legendre, and the domain of $T^{*}$ is $\nabla f(K)$, which is closed and convex by assumption. Applying Propositions 3.1 and 2.7(iii), we get that Fix $\left(T^{*}\right)=\nabla f(\operatorname{Fix}(T))$ is closed and convex, as asserted.

Nevertheless, it is not always possible to deduce results for right Bregman nonexpansive mappings by means of the conjugate operator. An example of this is Proposition 3.6, as it is pointed out in Remark 3.7.

Recall that a mapping $B: X \rightarrow X^{*}$ is said to be weakly sequentially continuous if the weak convergence of $\left\{x_{n}\right\}_{n \in \mathbb{N}} \subset X$ to $x$ implies the weak convergence of $\left\{B x_{n}\right\}_{n \in \mathbb{N}}$ to $B x$. The following result brings out a consequence of weak sequential continuity.

Proposition 3.5. Let $B: X \rightarrow X^{*}$ be a weakly sequentially continuous mapping. Then $B$ is bounded on bounded subsets of $\operatorname{dom} B$.

Proof. Let $K$ be a bounded subset of dom $B$. We have to show that $B(K)$ is bounded too. Suppose by the way of contradiction that $B(K)$ is not bounded. Then there is a sequence $\left\{x_{n}\right\}_{n \in \mathbb{N}}$ in $K$ such that $\lim _{n \rightarrow \infty}\left\|B\left(x_{n}\right)\right\|=+\infty$. On the other hand, there exists a subsequence $\left\{x_{n_{k}}\right\}_{k \in \mathbb{N}}$ which converges weakly to some $x$ as $k \rightarrow \infty$. From the weak sequential continuity of $B$ it follows that the sequence $\left\{B x_{n_{k}}\right\}_{k \in \mathbb{N}}$ converges weakly to $B x$. Hence this sequence is bounded. The contradiction we have reached shows that $B$ is indeed bounded on bounded subsets of $\operatorname{dom} B$, as asserted.

Next we show that if $f$ is Legendre and uniformly continuous on bounded subsets of $X, \nabla f$ is weakly sequentially continuous, and $T$ is an R-BFNE operator, then the fixed point set of $T$ coincides with the set of its asymptotic fixed points. This means that the operator $I-T$ has a certain demi-closedness property.

Proposition 3.6 (Asymptotic fixed point set of R-BFNE operators). Let $f: X \rightarrow \mathbb{R}$ be Legendre and uniformly continuous on bounded subsets of $X$, and let $\nabla f$ be weakly sequentially continuous. Let $K$ be a nonempty subset of $X$ and let $T: K \rightarrow X$ be an $R$-BFNE operator. Then Fix $(T)=\widehat{\operatorname{Fix}}(T)$. 
Proof. The inclusion Fix $(T) \subset \widehat{\mathrm{Fix}}(T)$ is obvious. To show that $\widehat{\operatorname{Fix}}(T) \subset \operatorname{Fix}(T)$, let $u \in \widehat{\operatorname{Fix}}(T)$ be given. Then there is a sequence $\left\{x_{n}\right\}_{n \in \mathbb{N}}$ in $K$ such that $x_{n} \rightarrow u$ and $x_{n}-T x_{n} \rightarrow 0$ as $n \rightarrow \infty$. Since $f$ is uniformly continuous on bounded subsets of $X$, it follows that $f\left(x_{n}\right)-f\left(T x_{n}\right) \rightarrow 0$ as $n \rightarrow \infty$. In addition, since $\nabla f$ is weakly sequentially continuous, $x_{n} \rightarrow u$ and $x_{n}-T x_{n} \rightarrow 0$ as $n \rightarrow \infty$, we know that $\nabla f\left(T x_{n}\right)-\nabla f(u) \rightarrow 0$ as $n \rightarrow \infty$. On the other hand, since $T$ is an R-BFNE operator, we have

$$
0 \leq D_{f}\left(u, T x_{n}\right)-D_{f}\left(T x_{n}, T u\right)+D_{f}\left(x_{n}, T u\right)-D_{f}\left(T u, T x_{n}\right) .
$$

From the three point identity (see (3)) and (32) we now obtain

$$
\begin{aligned}
D_{f}(T u, u) & =D_{f}\left(T u, T x_{n}\right)-D_{f}\left(u, T x_{n}\right)+\left\langle\nabla f\left(T x_{n}\right)-\nabla f(u), T u-u\right\rangle \\
& \leq D_{f}\left(x_{n}, T u\right)-D_{f}\left(T x_{n}, T u\right)+\left\langle\nabla f\left(T x_{n}\right)-\nabla f(u), T u-u\right\rangle \\
& =\left[f\left(x_{n}\right)-f(T u)-\left\langle\nabla f(T u), x_{n}-T u\right\rangle\right]- \\
& {\left[f\left(T x_{n}\right)-f(T u)-\left\langle\nabla f(T u), T x_{n}-T u\right\rangle\right] } \\
& +\left\langle\nabla f\left(T x_{n}\right)-\nabla f(u), T u-u\right\rangle \\
& =f\left(x_{n}\right)-f\left(T x_{n}\right)+\left\langle\nabla f(T u), T x_{n}-x_{n}\right\rangle \\
& +\left\langle\nabla f\left(T x_{n}\right)-\nabla f(u), T u-u\right\rangle .
\end{aligned}
$$

Since $f\left(x_{n}\right)-f\left(T x_{n}\right) \rightarrow 0, \nabla f\left(T x_{n}\right)-\nabla f(u) \rightarrow 0$, and $x_{n}-T x_{n} \rightarrow 0$ as $n \rightarrow \infty$, it follows that $D_{f}(T u, u) \leq 0$. Consequently, $D_{f}(T u, u)=0$ and from [3, Lemma 7.3(vi), page 642] it now follows that $T u=u$. That is, $u \in \operatorname{Fix}(T)$, as required.

Remark 3.7. Since an analogous result holds for L-BFNE operators ( $c f$. [35, Lemma 15.6, page 308]), it is natural to wonder whether Proposition 3.6 could be obtained by means of the conjugate operator $T^{*}$ (see Definition 2.6 and Proposition 2.7). In fact, to this end, according to the former result, we would need $f$ to be Legendre, and $f^{*}$ to be uniformly Fréchet differentiable and bounded on bounded subsets of int dom $f^{*}$. In addition, if we required $\nabla f$ to be uniformly continuous on bounded subsets of int $\operatorname{dom} f^{*}$, then it would follow from Proposition 2.7(iii) and (vi) that

$$
\widehat{\mathrm{Fix}}\left(T^{*}\right)=\nabla f(\widehat{\mathrm{Fix}}(T))
$$

and Fix $(T)=\widehat{\operatorname{Fix}}(T)$. However, note that these conditions are seemingly different from the ones required in Proposition 3.6.

Finally, we present a characterization of R-BFNE operators which emphasizes the strong connection between the nonexpansivity of $T$ and the monotonicity of $\nabla f \circ T$. 
Proposition 3.8 (Characterization of R-BFNE operators). Let $f: X \rightarrow \overline{\mathbb{R}}$ be an admissible function, $K \subset \operatorname{dom} f$ and $T: K \rightarrow \operatorname{int} \operatorname{dom} f$. Then $T$ is $R$-BFNE if and only if $\nabla f \circ T$ is $(I-T)$-monotone.

Proof. According to Definition 2.3(i*), $T$ is R-BFNE if and only if

$$
0 \leq\langle\nabla f(T x)-\nabla f(T y),(I-T) x-(I-T) y\rangle
$$

for all $x, y \in K$. Therefore $T$ is R-BFNE if and only if $\nabla f \circ T$ is $(I-T)$-monotone.

We use this proposition in Section 6 in our discussion of various examples of R-BFNE operators.

We refer the interested reader to [30] for a thorough discussion of R-BSNE operators.

\section{Right Bregman nonexpansive retractions}

Given two subsets $K \subset C \subset X$, an operator $R: C \rightarrow K$ is said to be a retraction of $C$ onto $K$ if $R x=x$ for each $x \in K$. A retraction $R: C \rightarrow K$ is said to be sunny (see $[26,32])$ if

$$
R(R x+t(x-R x))=R x
$$

for each $x \in C$ and any $t \geq 0$, whenever $R x+t(x-R x) \in C$.

Sunny nonexpansive retractions have been studied in several contexts; we mention, for example, extension problems for accretive operators, iterative methods for solving variational inequalities, and the convex feasibility problem. See, for instance, $[26,28]$ and the references therein.

In this section, we study sunny nonexpansive retractions with respect to Bregman distances. Our main aim is to provide a characterization of sunny R-QBNE retractions when $f$ is Legendre, totally convex and cofinite (see Theorem 4.5 below). To this end, the following proposition will be essential.

Proposition 4.1 (Characterization of R-QBFNE retractions). Let a totally convex $f: X \rightarrow \overline{\mathbb{R}}$ be Gâteaux differentiable in int dom $f \neq \emptyset$. Let $K \subset C \subset \operatorname{int} \operatorname{dom} f$ be two nonempty subsets. If $C$ is convex and $R$ is a retraction of $C$ onto $K$, then $R$ is both sunny and $R-Q B N E$ if and only if it is $R-Q B F N E$. 
Proof. First we assume that $R$ is a sunny R-QBNE operator. Let $x \in C$ and $y \in$ $K=\operatorname{Fix}(R)$. Denote $x_{t}:=R x+t(x-R x)$ for each $t \in[0,1]$. Since $R$ is a retraction and R-QBNE, we have

$$
D_{f}(R x, y)=D_{f}\left(R x_{t}, y\right) \leq D_{f}\left(x_{t}, y\right) .
$$

Thus

$$
D_{f}(R x, y)=\min \left\{D_{f}(z, y): z \in[x, R x]\right\}
$$

in other words, $R x=\overleftarrow{\operatorname{proj}_{[x, R x]}^{f}}(y)$. Using Proposition 2.1(ii), we see that

$$
0 \leq\left\langle\nabla f(R x)-\nabla f(y), x_{t}-R x\right\rangle
$$

for each $t \in[0,1]$. Setting $t=1$, we get

$$
0 \leq\langle\nabla f(R x)-\nabla f(y), x-R x\rangle
$$

for any $x \in C$ and $y \in K$, that is, $R$ is R-QBFNE.

Conversely, from the three point identity (see (3)), for any $x \in C$ and $y \in K=$ Fix $(R)$, we have

$$
D_{f}(x, y)=D_{f}(x, R x)+D_{f}(R x, y)+\langle\nabla f(R x)-\nabla f(y), x-R x\rangle .
$$

Hence, we obtain from (15) that

$$
D_{f}(x, y) \geq D_{f}(x, R x)+D_{f}(R x, y) \geq D_{f}(R x, y)
$$

for any $x \in C$ and $y \in \operatorname{Fix}(R)$. This means that $R$ is an R-QBNE operator. Now we prove that $R$ is sunny. To this end, for any $x \in C$ and $t>0$, set $x_{t}:=R x+t(x-R x)$. From (15) we have

$$
0 \leq\left\langle\nabla f\left(R x_{t}\right)-\nabla f(R x), x_{t}-R x_{t}\right\rangle
$$

and

$$
0 \leq\left\langle\nabla f(R x)-\nabla f\left(R x_{t}\right), x-R x\right\rangle
$$

Since $x_{t}-R x=t(x-R x)$, we get

$$
0 \leq t\left\langle\nabla f(R x)-\nabla f\left(R x_{t}\right), x-R x\right\rangle=\left\langle\nabla f(R x)-\nabla f\left(R x_{t}\right), x_{t}-R x\right\rangle
$$

and hence

$$
0 \leq\left\langle\nabla f\left(R x_{t}\right)-\nabla f(R x), R x-R x_{t}\right\rangle
$$

This implies that

$$
\left\langle\nabla f\left(R x_{t}\right)-\nabla f(R x), R x-R x_{t}\right\rangle=0 .
$$

Since $f$ is totally convex, $f$ is strictly convex, and therefore $\nabla f$ is strictly monotone. Thus $R x_{t}=R x$, that is, $R$ is sunny, as claimed. 
A nonempty subset $K \subset \operatorname{int} \operatorname{dom} f$ is said to be a right quasi-Bregman nonexpansive retract (sunny right quasi-Bregman nonexpansive retract) of $C$ if there exists an R-QBNE retraction (sunny R-QBNE retraction) $R$ of $C$ onto $K$, where $K \subset C \subset \operatorname{int} \operatorname{dom} f$.

Corollary 4.2. Let a totally convex $f: X \rightarrow \overline{\mathbb{R}}$ be Gâteaux differentiable in $\operatorname{int} \operatorname{dom} f$ $\neq \emptyset$. If $K \subset \operatorname{int} \operatorname{dom} f$ is a sunny $R$-QBNE retract of $C$, then the sunny $R-Q B N E$ retraction of $C$ onto $K$ is uniquely defined.

Proof. Assuming that there exist two sunny R-QBNE retractions $R$ and $S$ of $C$ onto $K$, we know by Proposition 4.1 that both these operators are R-QBFNE. Therefore, for any $x \in C$, since $R x, S x \in K$, we have

$$
\langle\nabla f(R x)-\nabla f(S x), x-R x\rangle \geq 0
$$

and

$$
\langle\nabla f(S x)-\nabla f(R x), x-S x\rangle \geq 0 .
$$

Thus

$$
\langle\nabla f(S x)-\nabla f(R x), S x-R x\rangle \leq 0,
$$

which implies that $S x=R x$ because $\nabla f$ is strictly monotone.

Under appropriate conditions on $f$, if $R$ is an R-QBNE retraction of $X$ onto $K$, then $\nabla f(K)$ is closed and convex.

Corollary 4.3. Let $f: X \rightarrow \mathbb{R}$ be Legendre and cofinite. If $K \subset X$ is an $R$-QBNE retract of $X$, then $\nabla f(K)$ is closed and convex in $X^{*}$.

Proof. Let $R: X \rightarrow K$ be an R-QBNE retraction. Then Fix $(R)=K$. Since $f$ takes values in $\mathbb{R}$, int $\operatorname{dom} f=X$, and hence it now follows from Proposition 3.3 that $\nabla f(K)$ is closed and convex.

We next prove that for any closed and convex set $K^{*} \subset X^{*}, \nabla f^{*}\left(K^{*}\right)$ is a sunny R-QBNE retract.

Proposition $4.4\left(\nabla f^{*}\left(K^{*}\right)\right.$ is a sunny R-QBNE retract). Let $f: X \rightarrow \mathbb{R}$ be Legendre. Assume that $f$ and $f^{*}$ are totally convex. Let $K^{*}$ be a nonempty, closed and convex subset of int dom $f^{*}$. Then the operator $R$ defined by $R=\nabla f^{*} \circ{\overleftarrow{\operatorname{proj}_{K}^{*}}}_{K^{*}}^{*} \circ \nabla f$ is a sunny $R$-QBNE retraction of $X$ onto $\nabla f^{*}\left(K^{*}\right)$. 
Proof. For any $x \in \nabla f^{*}\left(K^{*}\right)$, we have $\overleftarrow{\operatorname{proj}}_{K^{*}}^{f^{*}}(\nabla f(x))=\nabla f(x)$ because $\nabla f(x) \in$ $K^{*}$. This implies that

$$
R x=\left(\nabla f^{*} \circ \overleftarrow{\operatorname{proj}_{K}^{*}} f^{*} \circ \nabla f\right)(x)=\nabla f^{*}(\nabla f(x))=x
$$

for any $x \in \nabla f^{*}\left(K^{*}\right)$. Thus $R$ is onto $\nabla f^{*}\left(K^{*}\right)$ and $R x=x$ for all $x \in \nabla f^{*}\left(K^{*}\right)$, that is, $R$ is a retraction. Since $R$ is a retraction of $X$ onto $\nabla f^{*}\left(K^{*}\right)$, it follows that $\operatorname{Fix}(R)=\nabla f^{*}\left(K^{*}\right)$. Since $f^{*}$ is Gâteaux differentiable and totally convex, Proposition 2.1(iii), when applied to the left Bregman projection with respect to $f^{*}$ onto $K^{*}$, implies that

$$
D_{f^{*}}\left(\xi, \overleftarrow{\operatorname{proj}_{K^{*}}} f^{*}(\eta)\right)+D_{f^{*}}\left(\overleftarrow{\operatorname{proj}_{K^{*}}} f^{*}(\eta), \eta\right) \leq D_{f^{*}}(\xi, \eta)
$$

for all $\eta \in \operatorname{int} \operatorname{dom} f^{*}$ and $\xi \in K^{*}$. Therefore, for all $x \in X$ and $y \in \nabla f^{*}\left(K^{*}\right)$,

$$
\begin{aligned}
D_{f^{*}}\left(\nabla f(y), \overleftarrow{\operatorname{proj}_{K^{*}}^{*}} f^{*}(\nabla f(x))\right) & +D_{f^{*}}\left(\overleftarrow{\operatorname{proj}}_{K^{*}}^{f^{*}}(\nabla f(x)), \nabla f(x)\right) \\
& \leq D_{f^{*}}(\nabla f(y), \nabla f(x))
\end{aligned}
$$

or equivalently, by (26),

$$
D_{f}\left(\nabla f^{*} \circ \overleftarrow{\operatorname{proj}}_{K^{*}}^{f^{*}}(\nabla f(x)), y\right)+D_{f}\left(x, \nabla f^{*} \circ \overleftarrow{\operatorname{proj}}_{K^{*}}^{f^{*}}(\nabla f(x))\right) \leq D_{f}(x, y)
$$

That is,

$$
D_{f}(R x, y)+D_{f}(x, R x) \leq D_{f}(x, y)
$$

for all $x \in X$ and $y \in \nabla f^{*}\left(K^{*}\right)$. In other words, $R$ is R-QBFNE. It now follows from Proposition 4.1 that $R$ is a sunny R-QBNE retraction of $X$ onto $\nabla f^{*}\left(K^{*}\right)$.

The following theorem summarizes the previous results regarding sunny R-QBNE retractions. Earlier results in this spirit can be found in [14, Theorem 11.3, page 103] and [27, Theorem 3.3, page 203]. Recall that a mapping $B: X \rightarrow X^{*}$ is said to be demi-closed (weak-to-norm) if whenever a sequence $\left\{x_{n}\right\}_{n \in \mathbb{N}}$ converges weakly to $x$ and $B x_{n} \rightarrow \xi$, it follows that $B x=\xi$.

Theorem 4.5 (Characterization of sunny and R-QBNE retractions). Let $f: X \rightarrow \mathbb{R}$ be a Legendre, cofinite and totally convex function, and assume that $f^{*}$ is totally convex. Let $K$ be a nonempty subset of $X$. Then the following statements are equivalent.

(i) $K$ is a sunny $R$-QBNE retract of $X$. 
(ii) $K$ is an $R-Q B N E$ retract of $X$.

(iii) $\nabla f(K)$ is closed and convex.

Furthermore, if any of these statements holds, then $K$ is closed.

Proof. It is obvious that (i) implies (ii). From Corollary 4.3 it follows that (ii) implies (iii). Proposition 4.4 ensures that if $\nabla f(K)$ is closed and convex, then $R=$ $\nabla f^{*} \circ \overleftarrow{\operatorname{proj}}_{\nabla f(K)}^{f^{*}} \circ \nabla f$ is a sunny R-QBNE retraction of $X$ onto $K=\nabla f^{*}(\nabla f(K))$. Thus (iii) implies (i).

If any of these statements holds, $\nabla f(K)$ is closed and convex, so it is weakly closed. Since $\nabla f$ is norm-to-weak continuous, $\nabla f^{*}$ is demi-closed. Thus we get that $\nabla f^{*}(\nabla f(K))=K$ is closed.

Theorem 4.5, when combined with Corollary 4.2, shows that under the same assumptions, the unique sunny R-QBNE retraction of $X$ onto $K$ is given by the conjugate operator $\nabla f^{*} \circ \overleftarrow{\operatorname{proj}}_{\nabla f(K)}^{f^{*}} \circ \nabla f$, which is the right Bregman projection by (28). Thus we arrive at the following result.

Corollary 4.6. Let $f: X \rightarrow \mathbb{R}$ be a Legendre, cofinite and totally convex function, and assume that $f^{*}$ is totally convex. Let $K$ be a nonempty subset of $X$.

(i) If $\nabla f(K)$ is closed and convex, then the right Bregman projection,

$$
\overrightarrow{\operatorname{proj}}_{K}^{f}=\nabla f^{*} \circ \overleftarrow{\operatorname{proj}}_{\nabla f(K)}^{f^{*}} \circ \nabla f
$$

is the unique sunny $R$-QBNE retraction of $X$ onto $K$.

(ii) If $K$ is a sunny $R$-QBNE retract of $X$, then $\nabla f(K)$ is closed and convex, and $\overrightarrow{\operatorname{proj}}_{K}^{f}$ is the unique sunny $R$-QBNE retraction of $X$ onto $K$.

Now we are able to establish one of our most significant results, which guarantees that, under certain conditions on $f$, the fixed point set of any R-QBNE operator is a sunny R-QBNE retract of $X$ and, in particular, that the corresponding retraction is uniquely defined by the right Bregman projection onto the fixed point set, even if it is not necessarily closed or convex.

Corollary 4.7 ( Fix $(T)$ is a sunny R-QBNE retract). Let $f: X \rightarrow \mathbb{R}$ be a Legendre, cofinite and totally convex function, and assume that $f^{*}$ is totally convex. If $T: X \rightarrow$ $X$ is an $R$-QBNE operator, then there exists a unique sunny $R$-QBNE retraction of $X$ onto $\operatorname{Fix}(T)$, and this is the right Bregman projection onto Fix $(T)$. 
Proof. Since $f$ is Legendre and cofinite, and $T$ is R-QBNE, it follows from Proposition 3.3 that $\nabla f(\operatorname{Fix}(T))$ is closed and convex in $X^{*}$. Corollary 4.6(i) now ensures that the right Bregman projection is the unique sunny $\mathrm{R}$-QBNE retraction of $X$ onto $\operatorname{Fix}(T)$.

On the other hand, the left Bregman projection, $\overleftarrow{\operatorname{proj}}_{K}^{f}$, is not sunny, as can be seen in the following example.

Example 4.8 (Left Bregman projection is not sunny). Let $X=\mathbb{R}^{2}$ and consider the function $f\left(x_{1}, x_{2}\right)=x_{1} \ln \left(x_{1}\right)-x_{1}+x_{2} \ln \left(x_{2}\right)-x_{2}$ with $\operatorname{dom} f=(0,+\infty) \times(0,+\infty)$. Denote by $P_{K}$ the left Bregman projection onto the closed and convex set $K=$ $\left\{\left(y_{1}, y_{1}\right): 0<\alpha \leq y_{1}\right\}$. Calculations show that $P_{K}\left(x_{1}, x_{2}\right)=\left(\sqrt{x_{1} x_{2}}, \sqrt{x_{1} x_{2}}\right)$ when $x_{1} x_{2} \geq \alpha^{2}$. Hence it is clear that for any $t \in[0,2]$, we have

$$
\begin{aligned}
P_{K}\left(x_{1}, 4 x_{1}\right)+t\left(\left(x_{1}, 4 x_{1}\right)-P_{K}\left(x_{1}, 4 x_{1}\right)\right) & =2\left(x_{1}, x_{1}\right)+t\left(-x_{1}, 2 x_{1}\right) \\
& =\left((2-t) x_{1}, 2(1+t) x_{1}\right)
\end{aligned}
$$

and therefore for $t \neq 0,1$, we have

$$
2\left(x_{1}, x_{1}\right)=P_{K}\left(x_{1}, 4 x_{1}\right) \neq P_{K}\left((2-t) x_{1}, 2(1+t) x_{1}\right)=\sqrt{2-t} \sqrt{2} \sqrt{1+t}\left(x_{1}, x_{1}\right) .
$$

This shows that $P_{K}$ is not sunny.

Thanks to the relation between the right Bregman projection and the left Bregman projection it does have a sunny-like property.

Definition 4.9 ( $\nabla f$-sunny). Given $K \subset C \subset \operatorname{int} \operatorname{dom} f$, we say that a retraction $R$ of $C$ onto $K$ is $\nabla f$-sunny if for any $x \in C$, we have $R x_{t}=R x$ whenever $x_{t} \in C$, where $x_{t}=\nabla f^{*}(t \nabla f(x)+(1-t) \nabla f(R x)), t>0$.

Proposition 4.10 (Characterization of L-QBFNE operators). Let $f: X \rightarrow \overline{\mathbb{R}}$ be Legendre with $f^{*}$ totally convex and $K \subset \operatorname{int} \operatorname{dom} f$. If $R: \operatorname{int} \operatorname{dom} f \rightarrow K$ is a retraction, then $R$ is both $\nabla f$-sunny and $L-Q B N E$ if and only if $R$ is $L$-QBFNE.

Proof. Given a retraction $R: \operatorname{int} \operatorname{dom} f \rightarrow K$, we define the conjugate operator

$$
T^{*}:=\nabla f \circ R \circ \nabla f^{*}: \operatorname{int} \operatorname{dom} f^{*} \rightarrow \nabla f(K) \subset \operatorname{int} \operatorname{dom} f^{*} .
$$

It is easy to check that $T^{*}$ is a retraction of int dom $f^{*}$ onto $\nabla f(K)$. On the other hand, $R$ is $\nabla f$-sunny if and only if $T^{*}$ is sunny. Now Propositions 2.7 and 4.1 yield the desired equivalence. 
It follows from this result and Proposition 2.1 that the left Bregman projection $\overleftarrow{\operatorname{proj}}_{K}^{f}$ is an $\nabla f$-sunny L-QBNE retraction of int dom $f$ onto $K$.

Finally, we present an analogue of Proposition 2.1 (which concerns left Bregman projections).

Proposition 4.11 (Characterization of right Bregman projections). Let $f: X \rightarrow \mathbb{R}$ be a function such that $f^{*}$ is admissible and totally convex. Let $x \in X$ and let $K$ be a subset in int $\operatorname{dom} f$ such that $\nabla f(K)$ is closed and convex. If $\hat{x} \in K$, then the following conditions are equivalent.

(i) The vector $\hat{x}$ is the right Bregman projection of $x$ onto $K$ with respect to $f$.

(ii) The vector $\hat{x}$ is the unique solution of the variational inequality

$$
\langle\nabla f(z)-\nabla f(y), x-z\rangle \geq 0 \quad \forall y \in K
$$

(iii) The vector $\hat{x}$ is the unique solution of the inequality

$$
D_{f}(z, y)+D_{f}(x, z) \leq D_{f}(x, y) \quad \forall y \in K
$$

Proof. Since $\nabla f(K)$ is closed and convex, the left Bregman projection onto $\nabla f(K)$ with respect to the totally convex function $f^{*}$ is well defined and characterized in Proposition 2.1. It is clear from (28) that (i) is equivalent to the fact that the vector $\nabla f(\hat{x})$ is the left Bregman projection of $\nabla f(x)$ onto $\nabla f(K)$ with respect to $f^{*}$. Thence, from Proposition 2.1(ii), we get that (i) is equivalent to $\hat{x}$ being the unique solution of the inequality

$$
\left\langle\nabla f^{*}(\nabla f(x))-\nabla f^{*}(\nabla f(z)), \nabla f(z)-\xi\right\rangle \geq 0 \quad \forall \xi \in \nabla f(K) .
$$

But this is equivalent to

$$
\langle\nabla f(z)-\nabla f(y), x-z\rangle \geq 0 \quad \forall y \in K
$$

Using the three point identity (see (3)), we can also prove that (ii) is equivalent to (iii). 


\section{The conjugate resolvent}

We begin by recalling the definition and several basic properties of $\nabla f$-resolvents as established in [4, Proposition 3.8, page 604], where they were called $D$-resolvents. It is worth mentioning that this concept was generalized to $F$-resolvents in [7].

Definition 5.1 ( $\nabla f$-resolvent). Let $A: X \rightarrow 2^{X^{*}}$ be a set-valued mapping. The resolvent of $A$ with respect to $f$ is the operator $\operatorname{Res}_{A}^{f}: X \rightarrow 2^{X}$ which is defined by

$$
\operatorname{Res}_{A}^{f}:=(\nabla f+A)^{-1} \circ \nabla f .
$$

Proposition 5.2 (Properties of $\nabla f$-resolvents). Let $f: X \rightarrow \overline{\mathbb{R}}$ be an admissible function and let $A: X \rightarrow 2^{X^{*}}$ be an operator such that int $\operatorname{dom} f \cap \operatorname{dom} A \neq \emptyset$. The following statements hold.

(i) $\operatorname{dom}_{\operatorname{Res}_{A}^{f}} \subset \operatorname{int} \operatorname{dom} f$.

(ii) $\operatorname{ran} \operatorname{Res}_{A}^{f} \subset \operatorname{int} \operatorname{dom} f$.

(iii) Fix $\left(\operatorname{Res}_{A}^{f}\right)=\operatorname{int} \operatorname{dom} f \cap A^{-1}\left(0^{*}\right)$.

(iv) Suppose, in addition, that $A$ is a monotone mapping. Then the following assertions also hold.

(a) If $\left.f\right|_{\operatorname{int} \operatorname{dom} f}$ is strictly convex, then the operator $\operatorname{Res}_{A}^{f}$ is single-valued on its domain and L-BFNE.

(b) If $f: X \rightarrow \mathbb{R}$ is such that $\operatorname{ran} \nabla f \subset \operatorname{ran}(\nabla f+A)$, then $\operatorname{dom}_{\operatorname{Res}_{A}^{f}}=X$.

Motivated by Definition 2.6, we now define the conjugate $\nabla f$-resolvent in the dual space $X^{*}$.

Definition 5.3 (Conjugate $\nabla f$-resolvent). Let $A: X \rightarrow 2^{X^{*}}$ be a set-valued mapping. The conjugate resolvent of $A$ with respect to $f$, or the conjugate $\nabla f$-resolvent, is the operator $\operatorname{CRes}_{A}^{f}: X^{*} \rightarrow 2^{X^{*}}$ defined by

$$
\operatorname{CRes}_{A}^{f}:=\left(I+A \circ \nabla f^{*}\right)^{-1} .
$$

Indeed, the conjugate $\nabla f$-resolvent is the conjugate operator of the $\nabla f$-resolvent, as we show in the following lemma. 
Lemma 5.4. Let $A: X \rightarrow 2^{X^{*}}$ be a set-valued mapping and let $f: X \rightarrow \overline{\mathbb{R}}$ be an admissible function. Then

$$
\operatorname{CRes}_{A}^{f}=\nabla f \circ \operatorname{Res}_{A}^{f} \circ \nabla f^{*} .
$$

Thus, dom $\operatorname{CRes}_{A}^{f}=\nabla f\left(\operatorname{dom}_{\operatorname{Res}_{A}^{f}}^{f}\right)$ and $\operatorname{ran}_{\operatorname{CRes}_{A}^{f}}^{f}=\nabla f\left(\operatorname{ran}_{\operatorname{Res}_{A}^{f}}^{f}\right)$.

Proof. Indeed, since $(\nabla f)^{-1}=\nabla f^{*}$, it follows that

$$
\begin{aligned}
\nabla f \circ \operatorname{Res}_{A}^{f} \circ \nabla f^{*} & =\nabla f \circ\left((\nabla f+A)^{-1} \circ \nabla f\right) \circ \nabla f^{*} \\
& =\nabla f \circ(\nabla f+A)^{-1} \\
& =\left((\nabla f+A) \circ \nabla f^{*}\right)^{-1} \\
& =\left(I+A \circ \nabla f^{*}\right)^{-1} .
\end{aligned}
$$

The remaining statements follow from Proposition 2.7(i).

This connection between the $\nabla f$-resolvent and its conjugate allows us to deduce the following properties.

Proposition 5.5 (Properties of conjugate $\nabla f$-resolvents). Let $f: X \rightarrow \overline{\mathbb{R}}$ be an admissible function and let $A: X \rightarrow 2^{X^{*}}$ be a mapping such that int $\operatorname{dom} f \cap \operatorname{dom} A \neq$ $\emptyset$. The following statements hold.

(i) $\operatorname{dom} \operatorname{CRes}_{A}^{f} \subset \operatorname{int} \operatorname{dom} f^{*}$.

(ii) $\operatorname{ran} \operatorname{CRes}_{A}^{f} \subset \operatorname{int} \operatorname{dom} f^{*}$.

(iii) $\nabla f^{*}\left(\operatorname{Fix}\left(\operatorname{CRes}_{A}^{f}\right)\right)=\operatorname{int} \operatorname{dom} f \cap A^{-1}\left(0^{*}\right)$.

(iv) Suppose, in addition, that $A$ is a monotone mapping. Then the following assertions also hold.

(a) If $\left.f\right|_{\operatorname{int} \operatorname{dom} f}$ is strictly convex, then the operator $\operatorname{CRes}_{A}^{f}$ is single-valued on its domain and $R-B F N E$.

(b) If $f: X \rightarrow \mathbb{R}$ is such that $\operatorname{ran} \nabla f \subset \operatorname{ran}(\nabla f+A)$, then $\operatorname{dom}_{C^{2} e_{A}^{f}}^{f}=$ $\operatorname{int} \operatorname{dom} f^{*}$.

Proof. Basically all these properties are consequence of Propositions 2.7 and 5.2, because $\operatorname{CRes}_{A}^{f}$ is the conjugate operator of $\operatorname{Res}_{A}^{f}$ (see Lemma 5.4). 
The previous proposition provides sufficient conditions for the conjugate $\nabla f$ resolvent to be R-BFNE. Next we prove that to be the conjugate $\nabla f$-resolvent of a monotone mapping is also a necessary condition for an operator to be R-BFNE. See [7, Proposition 5.1, page 7] for the analogous result regarding L-BFNE operators.

Theorem 5.6 (Characterization of R-BFNE operators by means of the conjugate $\nabla f$-resolvent). Let $f: X \rightarrow \overline{\mathbb{R}}$ be an admissible function. Then the operator $T: K \subset$ $\operatorname{int} \operatorname{dom} f \rightarrow \operatorname{int} \operatorname{dom} f$ is $R$-BFNE if and only if the mapping $A: X \rightarrow 2^{X^{*}}$, defined by $A=\left(T^{-1}-I\right) \circ \nabla f$, is monotone.

Proof. By definition, it readily follows that $T=\operatorname{CRes}_{A}^{f}$. So the converse implication was already proved in Proposition 5.5(iv)(a). Now assume that $T$ is R-BFNE. In order to prove that $A$ is monotone, we denote the graph of $A$ by

$$
\operatorname{gra} A:=\left\{\left(z, z^{*}\right) \in X \times X^{*}: z^{*} \in A(z)\right\} .
$$

Then, for any $\left(z, z^{*}\right) \in$ gra $A$, we have

$$
\begin{aligned}
z^{*} \in A(z) & \Leftrightarrow z^{*} \in T^{-1}(\nabla f(z))-\nabla f(z) \\
& \Leftrightarrow T\left(z^{*}+\nabla f(z)\right)=\nabla f(z) \\
& \Leftrightarrow z=\nabla f^{*}\left(T\left(z^{*}+\nabla f(z)\right)\right) .
\end{aligned}
$$

Given $\left(x, x^{*}\right),\left(y, y^{*}\right) \in$ gra $A$, denote

$$
u^{*}=x^{*}+\nabla f(x) \text { and } v^{*}=y^{*}+\nabla f(y) .
$$

Since $T$ is R-BFNE, using equalities (35) and (36), we see that

$$
\begin{aligned}
\left\langle x-y, x^{*}-y^{*}\right\rangle & =\left\langle\nabla f^{*}\left(T u^{*}\right)-\nabla f^{*}\left(T v^{*}\right), x^{*}-y^{*}\right\rangle \\
& =\left\langle\nabla f^{*}\left(T u^{*}\right)-\nabla f^{*}\left(T v^{*}\right), u^{*}-v^{*}\right\rangle-\langle x-y, \nabla f(x)-\nabla f(y)\rangle \\
& \geq\left\langle x-y, T u^{*}-t v^{*}\right\rangle-\langle x-y, \nabla f(x)-\nabla f(y)\rangle \\
& =0
\end{aligned}
$$

which concludes the proof.

Note that in the case of a Hilbert space, if $f=(1 / 2)\|\cdot\|^{2}$, then both resolvents $\operatorname{Res}_{A}^{f}$ and $\operatorname{CRes}_{A}^{f}$ coincide with the classical resolvent

$$
\mathrm{R}_{A}:=(I+A)^{-1},
$$

and both Proposition 5.2 and Proposition 5.5, along with Theorem 5.6 and its left counterpart, recapture some of its well-known properties. 
Recall that, in the setting of Hilbert spaces, we know by Minty's Theorem [5, Theorem 21.1, page 311] (see also [23]), that under the assumption of monotonicity, if $A$ is maximal monotone, then $\mathrm{R}_{A}$ has full domain, and vice versa. In fact, Bauschke et al. [7] proved that, under suitable conditions on $f$, if $A$ is monotone, then $A$ is maximal monotone if and only if $\operatorname{ran}(A+\nabla f)=X^{*}$. On the one hand, since

$$
\operatorname{dom} \operatorname{Res}_{A}^{f}=\nabla f^{*}(\operatorname{ran}(A+\nabla f)),
$$

this result recovers Minty's Theorem in the framework of a Hilbert space. On the other hand, by Lemma 5.4 we know that $\operatorname{dom}_{\operatorname{CRes}_{A}^{f}}=\nabla f\left(\operatorname{dom}_{\operatorname{Res}_{A}^{f}}\right)$, that is,

$$
\operatorname{dom} \operatorname{CRes}_{A}^{f}=\operatorname{ran}(A+\nabla f)
$$

so the conjugate $\nabla f$-resolvent seems to be more appropriate for establishing a counterpart of Minty's Theorem in the context of the Bregman distance, resulting in the following characterization.

Theorem 5.7. Let $f: X \rightarrow \mathbb{R}$ be a strictly convex and cofinite admissible function, and let $A: X \rightarrow 2^{X^{*}}$ be a set-valued monotone mapping. Then $A$ is maximal monotone if and only if $\operatorname{dom}_{\mathrm{CRes}_{A}^{f}}=X^{*}$.

Next, we note that Corollary 4.7 and the fact that the conjugate $\nabla f$-resolvent of a monotone mapping is R-BFNE imply that the set $\nabla f\left(A^{-1}\left(0^{*}\right)\right)$ is a sunny R-QBNE retract of $X^{*}$.

Corollary 5.8. Let $f: X \rightarrow \mathbb{R}$ be Legendre, cofinite and totally convex, and assume that $\operatorname{ran} \nabla f \subset \operatorname{ran}(\nabla f+A)$. Let $A: X \rightarrow 2^{X^{*}}$ be a monotone mapping with $\operatorname{dom} A \neq \emptyset$. Then there exists a unique sunny $R$-QBNE retraction of $X$ onto $\nabla f\left(A^{-1}\left(0^{*}\right)\right)$, and this is the right Bregman projection onto $\nabla f\left(A^{-1}\left(0^{*}\right)\right)$.

Remark 5.9. Given a nonempty, closed and convex subset $K$ of $X$, let $A=\partial \iota_{K}$, where $\iota_{K}$ is the indicator function of $K$. Then, since we know that $\operatorname{Res}_{A}^{f}=\overleftarrow{\operatorname{proj}}_{K}^{f}$, it follows from Lemma 5.4 that

$$
\operatorname{CRes}_{A}^{f}=\nabla f \circ \overleftarrow{\operatorname{proj}}_{K}^{f} \circ \nabla f^{*}=\overrightarrow{\operatorname{proj}}_{\nabla f(K)}^{f^{*}}
$$

which, under suitable conditions on $f$, is the unique sunny R-QBNE retraction of $X$ onto $\nabla f(K)$. 


\section{Examples of R-BFNE operators}

In this section we use conjugation, $T$-monotonicity and the conjugate $\nabla f$-resolvent to present various examples of R-BFNE operators. Indeed, we have already seen that RBFNE operators can be generated from L-BFNE operators (Proposition 2.7), as well as from T-monotone (Proposition 3.8) and monotone mappings (Proposition 5.5). We begin with one-dimensional examples for several choices of the function $f$. These examples are followed by examples in infinite dimensional spaces constructed by means of the conjugate $\nabla f$-resolvents of the identity operator and the right Bregman projection, for a particular type of functions $f$.

\subsection{The case of the real line}

Assume that $X=\mathbb{R}$. In this case the notion of $T$-monotonicity can be simplified and a simpler characterization of R-BFNE operators can be obtained as follows.

Lemma 6.1. Let $f: \mathbb{R} \rightarrow \overline{\mathbb{R}}$ be a differentiable function and consider an operator $T: K \subset \operatorname{int} \operatorname{dom} f \rightarrow \mathbb{R}$. The functions $f^{\prime} \circ T$ and $I-T$ are increasing (decreasing) if and only if $T$ is $R$-BFNE. Therefore, whenever one of the following conditions holds, $T$ is $R$-BFNE.

(i) $f^{\prime}$ is increasing and

(a) $T$ and $I-T$ are increasing functions;

(b) in particular, $T$ is differentiable on int $\operatorname{dom} T$ and its derivative $T^{\prime}$ satisfies $0 \leq T^{\prime} \leq 1$

(ii) $f^{\prime}$ is decreasing and either $T$ or $I-T$ is decreasing.

Using Lemma 6.1, in Subsection 6.1.2 we generate examples of R-BFNE operators defined on the real line. An analogous argument has recently been used in [11] to produce L-BFNE operators with respect to the Boltzmann-Shannon entropy

$$
\mathcal{B S}(x):=x \log (x)-x, \quad 0<x<+\infty
$$

and the Fermi-Dirac entropy

$$
\mathcal{F D}(x):=x \log (x)+(1-x) \log (1-x), \quad 0<x<1,
$$

two important functions with many applications (see [11] and the references therein). Each of these two entropies is defined to be zero, by its limit, at the end points of their domains. 
Bearing in mind the definition of the conjugate operator $T^{*}$ and its properties in relation to the original operator $T$ (see Proposition 2.7), we may get examples of RBFNE operators by computing the conjugate operator of known L-BFNE operators. Indeed, we know that

$T$ is L-BFNE wrt $f$ on $K \subset \mathbb{R} \Leftrightarrow T^{*}$ is R-BFNE wrt $f^{*}$ on $f^{\prime}(K) \subset \mathbb{R}$.

In Table 2 we exhibit the conjugate operator of $T$ with respect to certain functions $f$. So, on the one hand, the existing examples of L-BFNE operators with respect to $f$ allow us to obtain R-BFNE operators with respect to $f^{*}$ (Subsection 6.1.1). On the other hand, by using the notion of $T$-monotonicity (Subsection 6.1.2), we provide examples of R-BFNE operators, which lead, in their turn, to new examples of L-BFNE operators.

\begin{tabular}{|c|c|c|c|}
\hline$f(x)$ & $f^{*}(x)$ & $T^{*}(x)$ & $\mathcal{D}^{*}$ \\
\hline \hline $\mathcal{B S}(x)$ & $e^{x}$ & $\log \left(T\left(e^{x}\right)\right)$ & $\mathbb{R}$ \\
\hline$e^{x}$ & $\mathcal{B S}(x)$ & $e^{T(\log x)}$ & $(0,+\infty)$ \\
\hline$x^{p} / p, p \in(0,+\infty)$ & $x^{q} / q$ & $T\left(x^{p-1}\right)^{q-1}$ & $\mathbb{R}$ \\
\hline $\mathcal{F D}(x)$ & $\log \left(1+e^{x}\right)$ & $\log \left(\frac{T\left(\frac{e^{x}}{1+e^{x}}\right)}{1-T\left(\frac{e^{x}}{1+e^{x}}\right)}\right)$ & $\mathbb{R}$ \\
\hline $\log \left(1+e^{x}\right)$ & $\mathcal{F} \mathcal{D}(x)$ & $\frac{e^{T\left(\log \left(\frac{x}{1-x}\right)\right)}}{1+e^{T\left(\log \left(\frac{x}{1-x}\right)\right)}}$ & $(0,1)$ \\
\hline$-\log x$ & $-1-\log (-x)$ & $-\frac{1}{T\left(\frac{1}{x}\right)}$ & $\mathbb{R} \backslash\{0\}$ \\
\hline$-1-\log (-x)$ & $-\log x$ & $\frac{1}{T\left(-\frac{1}{x}\right)}$ & $\mathbb{R} \backslash\{0\}$ \\
\hline
\end{tabular}

Table 2: Conjugate operators of $T$ with respect to $f$

Remark 6.2 (R-BFNE operators in $\mathbb{R}^{n}$ ). Denote $I=\{1,2, \ldots, n\}$. Let $f_{i}: \mathbb{R} \rightarrow \mathbb{R}$ $(\forall i \in I)$ be an admissible function, and define the function $\mathbf{F}: \mathbb{R}^{n} \rightarrow \mathbb{R}$ by

$$
\mathbf{F}\left(x_{1}, \ldots, x_{n}\right)=\sum_{i \in I} f_{i}\left(x_{i}\right)
$$


with int $\operatorname{dom} \mathbf{F}=\prod_{i \in I} \operatorname{int} \operatorname{dom} f_{i}$. Let $K_{i}(\forall i \in I)$ be a nonempty subset of int dom $f_{i}$ and $\mathbf{K}:=\prod_{i \in I} K_{i}$. We define the operator $\mathbf{T}: \mathbf{K} \rightarrow \operatorname{int} \operatorname{dom} \mathbf{F}$ by

$$
\mathbf{T}\left(x_{1}, \ldots, x_{n}\right)=\left(T_{1}\left(x_{1}\right), \ldots, T_{n}\left(x_{n}\right)\right),
$$

where $T_{i}: K_{i} \rightarrow \operatorname{int} \operatorname{dom} f_{i}(\forall i \in I)$. If each $T_{i}$ is R-BFNE with respect to $f_{i}$ on $K_{i}$, then it is easy to prove that the operator $\mathbf{T}$ is R-BFNE with respect to $\mathbf{F}$ on $\mathbf{K}$. $\diamond$

\subsubsection{Examples generated by conjugation}

By using the examples presented in [11, Example 4.14, page 174] and Table 2, and applying Proposition 2.7, we can provide more examples of R-BFNE operators. More precisely, Table 3 contains R-BFNE operators $T^{*}$ with respect to $f^{*}$ on $\mathcal{D}^{*}$, which are generated by conjugation of L-BFNE operators $T$ with respect to $f$ on $\mathcal{D}$.

\begin{tabular}{|c|c|c|c|c|c|}
\hline \multicolumn{3}{|c|}{ L-BFNE wrt $f$ on $\mathcal{D}$} & \multicolumn{3}{|c|}{ R-BFNE wrt $f^{*}$ on $\mathcal{D}^{*}$} \\
\hline$T(x)$ & $f(x)$ & $\mathcal{D}$ & $T^{*}(x)$ & $f^{*}(x)$ & $\mathcal{D}^{*}$ \\
\hline $\begin{array}{c}\alpha x+\beta \\
\alpha, \beta \in(0,+\infty)\end{array}$ & \multirow{5}{*}{$\mathcal{B S}(x)$} & $(0,+\infty)$ & $\log \left(\alpha e^{x}+\beta\right)$ & \multirow{5}{*}{$e^{x}$} & $\mathbb{R}$ \\
\hline $\begin{array}{c}x^{p} \\
p \in(0,1]\end{array}$ & & $(0,+\infty)$ & $p x$ & & $\mathbb{R}$ \\
\hline $\log x$ & & {$[e,+\infty)$} & $\log x$ & & {$[1,+\infty)$} \\
\hline$e^{x}$ & & $(0,1]$ & $e^{x}$ & & $(-\infty, 0)$ \\
\hline $\sin x$ & & $(0, \pi / 2]$ & $\log \left(\sin e^{x}\right)$ & & $(-\infty, \log (\pi / 2)]$ \\
\hline $\begin{array}{c}\alpha \\
\alpha \in(0,1)\end{array}$ & \multirow{3}{*}{$\mathcal{F D}(x)$} & $(0,1)$ & $\begin{array}{c}\alpha \\
\alpha \in \mathbb{R}\end{array}$ & \multirow{3}{*}{$\log \left(1+e^{x}\right)$} & $\mathbb{R}$ \\
\hline $\begin{array}{c}\alpha x \\
\alpha \in(0,1)\end{array}$ & & $(0,1)$ & $\log \left(\frac{\alpha e^{x}}{1+(1-\alpha) e^{x}}\right)$ & & $\mathbb{R}$ \\
\hline $\begin{array}{c}x^{p} \\
p \in(0,1)\end{array}$ & & $(0,1)$ & $\log \left(\left(\frac{1+e^{x}}{e^{x}}\right)+1\right)$ & & $\mathbb{R}$ \\
\hline
\end{tabular}

Table 3: R-BFNE operators generated by conjugation 


\subsubsection{Examples generated by $T$-monotonicity}

Using the connections with $T$-monotonicity (Lemma 6.1), one is able to prove easily that the operators $T$ shown in Table 4 are R-BFNE with respect to $f$ on $\mathcal{D}$. We

\begin{tabular}{|c|c|c|}
\hline$T(x)$ & $f(x)$ & $\mathcal{D}$ \\
\hline$\alpha x+\beta \quad \alpha \in[0,1], \beta \in \mathbb{R}$ & \multirow{4}{*}{$\mathcal{B S}(x)$} & $(0,+\infty)$ \\
\hline$x^{p} \quad p \in(0,1]$ & & $\left(0,(1 / p)^{1 /(p-1)}\right)$ \\
\hline$\alpha \log x \quad \alpha \in(0,+\infty)$ & & {$[\alpha,+\infty)$} \\
\hline $\cos x$ & & {$[\pi, 2 \pi]$} \\
\hline$\alpha e^{x} \quad \alpha \in \mathbb{R}$ & \multirow{3}{*}{$\log x$} & $(0,+\infty)$ \\
\hline $\sin x$ & & {$[\pi / 2,3 \pi / 2]$} \\
\hline $\cot x$ & & $(0, \pi]$ \\
\hline$\alpha x+\beta \quad \alpha \in[0,1], \beta \in \mathbb{R}$ & \multirow{4}{*}{$\mathcal{F D}(x)$} & $(0,1)$ \\
\hline$x^{p} \quad p \in(0,1]$ & & $\left(0,(1 / p)^{1 /(p-1)}\right)$ \\
\hline$\alpha \log x \quad \alpha \in(0,+\infty)$ & & {$[\alpha, 1)$} \\
\hline $\sin x$ & & $(0,1)$ \\
\hline$\alpha x+\beta \quad \alpha \in[0,1], \beta \in \mathbb{R}$ & \multirow{3}{*}{$e^{x}$} & $\mathbb{R}$ \\
\hline$e^{x}$ & & $(-\infty, 0)$ \\
\hline $\log x$ & & {$[1,+\infty)$} \\
\hline
\end{tabular}

Table 4: R-BFNE operators wrt $f$ on $\mathcal{D}$ generated by monotonicity

provide another example which requires a bit more details.

Example 6.3. The Lambert function, $W$, is defined to be the inverse of $x \mapsto x e^{x}$. Its principal branch on the real axis is concave and increasing, and its domain is $(-1 / e,+\infty)(c f .[10,12])$. Then $T(x)=W(x)$ is R-BFNE with respect to $\mathcal{B S}$ and $e^{x}$ on $(0,+\infty)$ and with respect to $\mathcal{F D}$ on $(0,1)$.

Proof. Given $x \in(-1 / e,+\infty)$, we compute $T^{\prime}(x)$. Set $W(x)=y$, which means that $y e^{y}=x$. Then we differentiate both sides and get that $y^{\prime} e^{y}+y y^{\prime} e^{y}=1$. Since 
$y e^{y}=x$, we obtain that $y^{\prime}=1 /\left(x+e^{y}\right)$. Therefore, as $e^{y}=x / y=x / W(x)$, it follows that

$$
W^{\prime}(x)=\frac{W(x)}{x+x W(x)} .
$$

Since

$$
\frac{W(x)}{x+x W(x)}=\frac{y}{y e^{y}+y^{2} e^{y}}=\frac{1}{e^{y}+y e^{y}},
$$

it is easy to see that $0<T^{\prime}(x) \leq 1$ if $y \geq 0$, that is, if $W(x) \geq 0$ and this happens when $x \geq 0$. Therefore, since the derivatives $\mathcal{B S}^{\prime}$ and $\mathcal{F} \mathcal{D}^{\prime}$ are increasing functions, it follows from Lemma 6.1(i)(b) that $T(x)=W(x)$ is a R-BFNE with respect to $\mathcal{B S}$ and $e^{x}$ on any nonempty subset of $(0,+\infty)$, and with respect to $\mathcal{F} \mathcal{D}$ on any nonempty subset of $(0,1)$.

\subsubsection{Examples of conjugate $\nabla f$-resolvents}

Example 6.4. Let $f(x)=\mathcal{B S}(x)$. Recall that $f^{*}(y)=e^{y}$. Thus, for any operator $A: \mathbb{R} \rightarrow \mathbb{R}$, the conjugate $\mathcal{B S}$-resolvent is

$$
\operatorname{CRes}_{A}^{\mathcal{B S}}(x)=y, \quad \text { with } y+A\left(e^{y}\right)=x .
$$

\begin{tabular}{|c|c|c|}
\hline$A(x)$ & $f(x)$ & $\operatorname{CRes}_{A}^{f}(x)$ \\
\hline$\alpha \quad \alpha \in(-1 / 2,+\infty)$ & \multirow{4}{*}{$\mathcal{B S}(x)$} & $\log \left(\frac{1}{\alpha} W\left(\alpha e^{x}\right)\right)$ \\
\hline$\alpha x+\beta \quad \alpha \in(0,+\infty), \beta \in \mathbb{R}$ & & $\log \left(\frac{1}{\alpha} W\left(\alpha e^{x-\beta}\right)\right)$ \\
\hline$\alpha \log x \quad \alpha \neq-1$ & & $\frac{x}{1+\alpha}$ \\
\hline$\frac{x^{p}}{p} \quad p \in \mathbb{R} \backslash\{0\}$ & & $\frac{1}{p} \log \left(W\left(e^{p x}\right)\right)$ \\
\hline
\end{tabular}

Using this, one is able to compute $\operatorname{CRes}_{A}^{\mathcal{B S}}$ for different choices of the operator $A$. See Table 5, where $W$ stands for the Lambert function.

Table 5: Conjugate $\nabla f$-resolvents of $A$

\subsection{The infinite dimensional case}

\subsubsection{The right Bregman projection with respect to $\|\cdot\|^{p}$}

In uniformly convex and smooth Banach spaces, the left Bregman projection onto a half-space with respect to $f_{p}:=\|\cdot\|^{p}, p \in(1,+\infty)$, was computed in [19]. 
Proposition 6.5 (Left Bregman projection onto a hyperplane). Let $X$ be a uniformly convex and smooth Banach space and let

$$
K=\{z \in X:\langle a, z\rangle=b\},
$$

where $a \in X^{*} \backslash\{0\}$ and $b \in \mathbb{R}$. For any $x \in X$ and for all $p \in(1,+\infty)$, the following statements hold.

(i) The equation

$$
\left\langle a, \nabla f_{p}^{*}\left(s a+\nabla f_{p}(x)\right)\right\rangle=b
$$

has solutions $s$ such that $\operatorname{sign} s=\operatorname{sign} b-\langle a, x\rangle$.

(ii) The left Bregman projection (4) with respect to $f_{p}$ is given by

$$
\overleftarrow{\operatorname{proj}_{K}^{f}} f_{p}(x)=\nabla f_{p}^{*}\left(s a+\nabla f_{p}(x)\right)
$$

where $s \in \mathbb{R}$ is a solution of (39).

(iii) Formula (40) remains true when $K$ is the half-space $\{z \in X:\langle a, z\rangle \geq b\}$ and $s$ is a nonnegative solution of (39).

This result allows us to get a nice formula for the right Bregman projection onto $\nabla f_{p}(K)$ with respect to $f_{p}^{*}$, for any half-space $K$ in $X$. Indeed, we know from (28) that

$$
\overrightarrow{\operatorname{proj}}_{\nabla f(K)}^{f^{*}}=\nabla f \circ \overleftarrow{\operatorname{proj}}_{K}^{f} \circ \nabla f^{*}
$$

Then, from Proposition 6.5(ii), we have, for any $\xi \in X^{*}$,

$$
\overrightarrow{\operatorname{proj}}_{\nabla f_{p}(K)}^{*}(\xi)=\left(\nabla f_{p} \circ \overleftarrow{\operatorname{proj}_{K}^{f}} f_{p} \circ \nabla f_{p}^{*}\right)(\xi)=\left(s a+\nabla f_{p}\left(\nabla f_{p}^{*}(\xi)\right)\right)=s a+\xi,
$$

where $s \in \mathbb{R}$ is a solution of (39).

\subsubsection{The conjugate $\frac{1}{p}\|\cdot\|^{p}$-resolvent of the identity operator}

Following [7, Example 9.6], we consider the function $f: H \rightarrow \mathbb{R}, f(x)=\frac{1}{p}\|x\|^{p}$, where $H$ is a Hilbert space and $p \in(1,+\infty)$. So the conjugate of $f$ is the function $f^{*}(y)=\frac{1}{q}\|y\|^{q}$, where $q$ is the conjugate exponent of $p$, that is, $\frac{1}{p}+\frac{1}{q}=1$. Then, for any $y \neq 0, \nabla f^{*}(y)=\|y\|^{q-2} y$. Consider $A=I$, the identity operator, and denote the conjugate $\nabla f$-resolvent of $A$ by

$$
T_{q}=\operatorname{CRes}_{A}^{f}:=\left(I+\nabla f^{*}\right)^{-1} .
$$


Then

$$
T_{q}(x)= \begin{cases}0 & \text { if } x=0 \\ \alpha(x) x & \text { if } x \neq 0\end{cases}
$$

where $\alpha(x) \in(0,1)$ is the unique solution to the equation

$$
\alpha+\alpha^{q-1}\|x\|^{q-2}=1
$$

Indeed, given $x \in H$,

$$
T_{q}(x)=y \Leftrightarrow y+\nabla f^{*}(y)=x \Leftrightarrow y\left(1+\|y\|^{q-2}\right)=x .
$$

So we can write $y=\alpha(x) x$, where $\alpha(x) \in(0,1)$ solves equation (42). It is easy to check that this equation indeed has a unique solution in $(0,1)$.

Acknowledgements. The first author was partially supported by DGES (Grant BFM2009-1096-C02-01) and by the Junta de Andalucía (Grant FQM-127); the second and third authors were partially supported by the Israel Science Foundation (Grant 647/07), the Graduate School of the Technion, the Fund for the Promotion of Research at the Technion and by the Technion VPR Fund. Part of the research pertaining to this paper was carried out when the authors were visiting each other's institutions. They thank their colleagues for their kind hospitality. All the authors are very grateful to an anonymous referee for a detailed and insightful report, with many helpful comments and suggestions.

\section{References}

[1] Aoyama, K., Kohsaka, F. and Takahashi, W.: Three generalizations of firmly nonexpansive operators: their relations and continuity properties, J. Nonlinear Convex Anal. 10 (2009), 131-147.

[2] Bauschke, H. H. and Borwein, J. M.: Legendre functions and the method of random Bregman projections, J. Convex Anal. 4 (1997), 27-67.

[3] Bauschke, H. H., Borwein, J. M. and Combettes, P. L.: Essential smoothness, essential strict convexity, and Legendre functions in Banach spaces, Comm. Contemp. Math. 3 (2001), 615-647.

[4] Bauschke, H. H., Borwein, J. M. and Combettes, P. L.: Bregman monotone optimization algorithms, SIAM J. Control Optim. 42 (2003), 596636. 
[5] Bauschke, H. H. and Combettes, P. L. : Convex Analysis and Monotone Operator Theory in Hilbert Spaces, Springer, New York, 2011.

[6] Bauschke, H. H., Macklem, M. S. and Wang, X.: Chebyshev sets, Klee sets, and Chebyshev centers with respect to Bregman distances: recent results and open problems, Fixed-Point Algorithms for Inverse Problems in Science and Engineering, Optimization and Its Applications, Springer, New York, 2011, 1-21.

[7] Bauschke, H. H., Wang, X. and Yao, L.: General resolvents for monotone operators: characterization and extension, Biomedical Mathematics: Promising Directions in Imaging, Therapy Planning and Inverse Problems, Medical Physics Publishing, Madison, WI, USA, 2009, 57-74.

[8] Bauschke, H. H., Wang, X., Ye, J. and Yuan, X.: Bregman distances and Chebyshev sets, J. Approx. Theory 159 (2009), 3-25.

[9] Bonnans, J. F. and Shapiro, A.: Perturbation Analysis of Optimization Problems, Springer, New York, 2000.

[10] Borwein, J. M. and Lewis, A. S.: Convex Analysis and Nonlinear Optimization. Theory and Examples, 2nd Edition, Springer, New York, 2006.

[11] Borwein, J. M., Reich, S. and Sabach, S.: A characterization of Bregman firmly nonexpansive operators using a new monotonicity concept, J. Nonlinear Convex Anal. 12 (2011), 161-184.

[12] Borwein, J. M. and Vanderwerff, J.: Convex Functions: Constructions, Characterizations and Counterexamples, Encyclopedia of Mathematics and its Applications, Cambridge University Press, Cambridge, 2010.

[13] Bregman, L. M.: The relaxation method of finding the common point of convex sets and its application to the solution of problems in convex programming, USSR Comput. Math. and Math. Phys. 7 (1967), 200-217.

[14] Bruck, R. E.: Approximating Fixed Points and Fixed Point Sets of Nonexpansive Mappings in Banach Spaces, Ph. D. Thesis, The University of Chicago, 1969.

[15] Bruck, R. E.: Nonexpansive projections on subsets of Banach spaces, Pacific J. Math. 47 (1973), 341-355. 
[16] Bruck, R. E. and Reich, S.: Nonexpansive projections and resolvents of accretive operators in Banach spaces, Houston J. Math. 3 (1977), 459470.

[17] Butnariu, D., Censor, Y. and Reich, S.: Iterative averaging of entropic projections for solving stochastic convex feasibility problems, Comput. Optim. Appl. 8 (1997), 21-39.

[18] Butnariu, D. and Iusem, A. N.: Totally Convex Functions for Fixed Points Computation and Infinite Dimensional Optimization, Kluwer Academic Publishers, Dordrecht, 2000.

[19] Butnariu, D. and Resmerita, E.: Bregman distances, totally convex functions and a method for solving operator equations in Banach spaces, Abstr. Appl. Anal. 2006 (2006), Art. ID 84919, 1-39.

[20] Censor, Y. and Lent, A.: An iterative row-action method for interval convex programming, J. Optim. Theory Appl. 34 (1981), 321-353.

[21] Censor, Y. and Zenios, S. A.: Parallel Optimization, Oxford University Press, New York, 1997.

[22] Dotson, W. G., Jr.: Fixed points of quasi-nonexpansive mappings, J. Austral. Math. Soc. 13 (1972), 167-170.

[23] Eckstein, J. and Bertsekas, D. P.: On the Douglas-Rachford splitting method and the proximal point algorithm for maximal monotone operators, Math. Programming 55 (1992), 293-318.

[24] Goebel, K. and Kirk, W. A.: Topics in Metric Fixed Point Theory, Cambridge Studies in Advanced Mathematics, vol. 28, Cambridge University Press, Cambridge, 1990.

[25] Goebel, K. and Kirk, W. A.: Classical theory of nonexpansive mappings, Handbook of Metric Fixed Point Theory, Kluwer Academic Publishers, Dordrecht, 2001, 49-91.

[26] Goebel, K. and Reich, S.: Uniform Convexity, Hyperbolic Geometry, and Nonexpansive Mappings, Marcel Dekker, New York and Basel, 1984.

[27] Kohsaka, F. and Takahashi, W.: Generalized nonexpansive retractions and a proximal-type algorithm in Banach spaces, J. Nonlinear Convex Anal. 8 (2007), 197-209. 
[28] Kopecká, E. and Reich, S.: Nonexpansive retracts in Banach spaces, Banach Center Publ. 77 (2007), 161-174.

[29] Martín-Márquez, V., Reich, S. and Sabach, S.: Iterative methods for approximating fixed points of Bregman nonexpansive operators, Discrete and Continuous Dynamical Systems, accepted for publication.

[30] Martín-Márquez, V., Reich, S. and Sabach, S.: Bregman strongly nonexpansive operators in Banach spaces, in preparation.

[31] Phelps, R. R.: Convex Functions, Monotone Operators, and Differentiability, 2nd Edition, Lecture Notes in Mathematics, vol. 1364, Springer, Berlin, 1993.

[32] Reich, S.: Asymptotic behavior of contractions in Banach spaces, $J$. Math. Anal. Appl. 44 (1973), 57-70.

[33] Reich, S.: Extension problems for accretive sets in Banach spaces, $J$. Func. Anal. 26 (1977), 378-395.

[34] Reich, S.: A weak convergence theorem for the alternating method with Bregman distances, Theory and Applications of Nonlinear Operators of Accretive and Monotone Type, Marcel Dekker, New York, 1996, 313-318.

[35] Reich, S. and Sabach, S.: Existence and approximation of fixed points of Bregman firmly nonexpansive operators in reflexive Banach spaces, Fixed-Point Algorithms for Inverse Problems in Science and Engineering, Optimization and Its Applications, vol. 49, Springer, New York, 2011, 301-316. 